\title{
Triangulation of 2-loop MHV amplituhedron from sign flips
}

\author{
Ryota Kojima \\ Department of Particle and Nuclear Physics, \\ SOKENDAI (The Graduate University for Advanced Studies), \\ Tsukuba, Ibaraki, 305-0801, Japan \\ KEK Theory Center, \\ Tsukuba, Ibaraki, 305-0801, Japan \\ E-mail: ryota@post.kek.jp
}

ABSTRACT: In this paper, we consider the triangulation of the 2-loop MHV amplituhedron from "sign flip" definition. Using the isomorphism between the $m=2, k=2$ tree amplituhedron and the 1-loop MHV physical amplituhedron, we found the direct triangulation of the 2-loop MHV amplituhedron from sign flips. This triangulation is different from the BCFW triangulation because of the structure of the cells. And we also found a formula of the canonical form of the $n$-point 2-loop MHV amplituhedron. This formula looks like a 2-loop version of the Kermit representation of the 1-loop MHV amplitude. We checked that the sum of these cells is consistent with the BCFW up to at least 22-pt numerically.

KEYwords: Scattering Amplitudes, Supersymmetric Gauge Theory

ARXIV EPRINT: 1812.01822 


\section{Contents}

1 The amplituhedron and triangulation 1

2 BCFW and double pentagon expansions 4

3 Triangulation of 2-loop MHV amplituhedron from sign flips 5

$\begin{array}{lll}3.1 & \text { Four and five point amplitudes } & 7\end{array}$

3.2 n-point amplitude 11

4 More 2-loop objects $\quad 13$

4.1 Log of the 2-loop MHV amplitude 13

$\begin{array}{ll}4.2 & \text { Square of the amplituhedron and positivity } \\ \end{array}$

$\begin{array}{llr}5 & \text { Conclusion } & 17\end{array}$

A Explicit calculation of the 2-loop 5-point MHV amplitude 18

B Explicit results of the 2-loop n-point MHV amplituhedron 27

C Explicit results of the 2-loop n-point MHV log amplituhedron 30

\section{The amplituhedron and triangulation}

Recent years an unexpected connection between the scattering amplitudes and new geometric structure have been revealed [1-4]. In planar $\mathcal{N}=4$ SYM theory, the amplituhedron gives a definition of the scattering amplitude in a purely geometric way $[5,6]$ and it has been explored from a variety of perspectives in the past few years (see e.g. [14-24]). The amplituhedron is a geometric object that conjectured to give the scattering amplitude in planar $\mathcal{N}=4 \mathrm{SYM}$ theory as its form related to the volume: the canonical form. The canonical form is defined that it has logarithmic singularities on all the boundaries of the amplituhedron [4].

There are two definitions of the amplituhedron, a generalization of the interior of plane polygons into the Grassmannian [5] and a topological definition "sign flip definition" [7]. To see the first definition, we consider a convex $n$-polygon in projective space. The vertices of this polygon can be represented as 3 -vectors $Z_{a}^{I}$ for $I=1,2,3$ and $a=1,2, \cdots, n$. The convexity means that all ordered minors $\left\langle Z_{a} Z_{b} Z_{c}\right\rangle>0$ for $a<b<c$ are positive. Then the interior of this polygon can be thought as the set of points $Y^{I}$ that $Y^{I}=c_{a} Z_{a}^{I}$ with $c_{a}>0$. 
The tree amplituhedron $\mathcal{A}_{m, k, n}$ can be obtained as the generalization of this structure into the Grassmannian

$$
Y_{\alpha}^{I}=C_{\alpha a} Z_{a}^{I}, \quad \text { for } \quad I=1, \cdots, k+m, \quad a=1, \cdots, n
$$

where $C$ is the positive Grassmannian $G_{+}(k, n)$ and $Z$ is the positive external data

$$
\left\langle Z_{a_{1}}, \cdots, Z_{a_{k+m}}\right\rangle>0 \text { for } a_{1}, \cdots<a_{k+m} .
$$

We can generalize this tree amplituhedron into the loop amplituhedron. We fix $m=4$, then $Y$ is the $k$-plane in $\mathrm{k}+4$ dimensional space. Let's consider $L 2$-dimensional planes $\mathcal{L}_{(i)}$ in 4-dimensional space complement of $Y$. These $\mathcal{L}$ are the different linear combination of the external data $\mathcal{Z}$

$$
\mathcal{L}_{(i) \alpha}^{I}=D_{a \alpha(i)} Z_{a}^{I}
$$

where $\mathrm{D}$ is the positive Grassmannian $G_{+}(2, n)$. Then the full amplituhedron $\mathcal{A}_{n, k, l}$ is the space of all $Y, \mathcal{L}_{(i)}$ of the form

$$
Y_{\alpha}^{I}=C_{\alpha a} Z_{a}^{I}, \quad \mathcal{L}_{(i) \alpha}^{I}=D_{a \alpha(i)} Z_{a}^{I}
$$

where all ordered minors of the matrix

$$
\left(\begin{array}{c}
D_{\left(i_{1}\right)} \\
D_{\left(i_{2}\right)} \\
\vdots \\
D_{\left(i_{l}\right)} \\
C
\end{array}\right)
$$

are positive.

Next, we consider the topological definition. Polytopes can be defined in two different ways. The first is called "vertex-centered": the polytopes is defined as the convex full of the points $Z_{a}^{I}$. This related to the " $Y^{I}=C_{a} Z_{a}^{I}$ " description of the amplituhedron. Second is a "face-centered" description of the polytope: we can obtain the polytope by the collection of inequalities associated with the facet of the polytope. This picture is generalized to the amplituhedron [7]. In addition to the boundary inequalities, sign flip characterization is needed in the case of the amplituhedron. The face-centered definition of the $m=2$ tree amplituhedron is

$Y$ is in the $m=2$ amplituhedron iff $\langle Y i i+1\rangle>0$ and the sequence $\{\langle Y 12\rangle, \cdots,\langle Y 1 n\rangle\}$ has precisely $k$ sign flips.

We can define $m=4$ amplituhedron similarly. The sign flip definition of the loop amplituhedron is

$$
\begin{aligned}
& \left\langle(Y A B)_{\gamma} i i+1\right\rangle>0,\langle Y i i+1 j j+1\rangle>0 \\
& \left\{\left(\langle Y A B)_{\gamma} 12\right\rangle, \cdots,\left\langle(Y A B)_{\gamma} 1 n\right\rangle\right\} \text { has } k+2 \text { sign flips } \\
& \langle Y 1234\rangle>0, \cdots,\langle Y 123 n\rangle \text { has } k \text { sign flips }
\end{aligned}
$$

where $\gamma$ is the number of loops. 
To obtain the scattering amplitude from this geometry, we need to consider how to obtain the canonical form from this geometry. There are several ways: first is to obtain the canonical form directly from the geometry [8-10]. Another way is to triangulate the amplituhedron into a more simple one that its form is trivial. The triangulation is related to the recursion relation of scattering amplitude.

Note that the $Y=C \cdot Z$ description of the amplituhedron is highly redundant. The space of $C_{\alpha a}$ is $k(n-k)$ dimensional and it is always larger than the dimension of the tree amplituhedron $k \times m$. Non-redundant map into $Y$ can only come from the $k \times m$ dimensional cells of $C \in G_{+}(k, n)$. It is difficult to obtain the canonical form of this highly redundant space directly, however, we can triangulate the amplituhedron into the nonredundant cells. It is straightforward to obtain the canonical form of this non-redundant cells; for example, the case of a triangle in the projective plane or the $n$-simplex in the $n$-dimensional projective space [5]. Then we can obtain the canonical form of this nonredundant cell and the full form is obtained from the sum of these forms.

One of the important example of the triangulation of the amplituhedron is the BCFW triangulation. In planar $\mathcal{N}=4 \mathrm{SYM}$, all loop order amplitudes can be obtained from the $\mathrm{BCFW}$ recursion relation [11] and there is a triangulation of the amplituhedron related to this BCFW recursion relation [3, 5, 24]. Another example is the direct triangulation. In the simple case, we can triangulate the amplituhedron directly from the geometry [2]. In the case of the polygon: $\mathcal{A}(k=1, m=2, n)$, the dimension of non-redundant cells is 2 and these cells are the triangle in the projective plane. Then the triangulation of the $\mathcal{A}(1,2, n)$ is straightforward from the geometry. Another example is the 1-loop MHV amplituhedron $\mathcal{A}(l=1, k=0, n)$. One of the triangulation of this case is known: Kermit representation

$$
M_{\mathrm{MHV}}^{1 \text {-loop }}=\sum_{i<j}[1 i i+1,1 j j+1]
$$

where

$$
[1 i i+1,1 j j+1]=\frac{\langle A B(1 i i+1) \cap(i j j+1)\rangle^{2}}{\langle A B 1 i\rangle\langle A B 1 i+1\rangle\langle A B i i+1\rangle\langle A B 1 j\rangle\langle A B 1 j+1\rangle\langle A B j j+1\rangle} .
$$

The dimension of each cell is 4 , then these cells are non-redundant cells. This triangulation is obtained directly from the geometry [2]. However, the more general case, it is difficult to obtain the triangulation directly from the geometry. To see this, we consider the 2loop n-point MHV amplituhedron. In the $Y=C \cdot Z$ description we have many positivity conditions that

$$
\text { All ordered minor of }\left(\begin{array}{c}
D_{(1)} \\
D_{(2)}
\end{array}\right) \text { are positive. }
$$

where $D_{(i)} \in G_{+}(2, n)$. Because of these positivity conditions, it is difficult to obtain the triangulation for general $n$-point 2-loop amplitude directly from the geometry in this $Y=C \cdot Z$ description. Of course, we can triangulate this from the BCFW approach and obtain the canonical form. For example, in the case of the 4-point, it begins with the $k=2, n=8$ tree amplitude and after taking two forward limits, we can obtain the 2-loop 
MHV amplitude. Then the question is that is it possible to obtain the triangulation of the 2-loop MHV amplituhedron directly from the geometry, without having to refer to any tree amplitude or the BCFW recursion relation?

In this paper, we consider the triangulation of the $n$-point 2-loop MHV amplituhedron. we will give the triangulation of this amplituhedron from the sign flip definition and the full form of this amplituhedron as the sum of these cells. This triangulation is obtained directly from the geometry, without having to refer to any tree amplitude or the BCFW recursion relation. Then we will compare with the $\mathrm{BCFW}$, local representation and our results.

\section{BCFW and double pentagon expansions}

First we review briefly the BCFW recursion relation and double pentagon representation of the $n$-point 2-loop MHV amplitude. The BCFW recursion relation for all loop amplitudes in planar $\mathcal{N}=4 \mathrm{SYM}[11]$ is

$$
\begin{aligned}
M_{n, k, l}(1, \cdots, n) & =M_{n-1, k, l}(1, \cdots, n-1) \\
& +\sum_{n_{L}, k_{L}, l_{L} ; j}[j j+1 n-1 n 1] M_{n_{R}, k_{R}, l_{R}}^{R}\left(1, \cdots, j, I_{j}\right) \times M_{n_{L}, k_{L}, l_{L}}^{L}\left(I_{j}, j+1, \cdots, \hat{n}_{j}\right) \\
& +\int_{\mathrm{GL}(2)}[A B n-1 n 1] \times M_{n+2, k+1, l-1}\left(1, \cdots, \hat{n}_{A B}, \hat{A}, B\right)
\end{aligned}
$$

where $n_{L}+n_{R}=n, k_{L}+k_{R}=k, l_{L}+l_{R}=l$ and

$$
\begin{aligned}
{[a, b, c, d, e] } & =\frac{\delta^{0 \mid 4}\left(\eta_{a}\langle b c d e\rangle+\text { cyclic }\right)}{\langle a b c d\rangle\langle b c d e\rangle\langle c d e a\rangle\langle\text { deab }\rangle\langle e a b c\rangle} & & \\
\hat{n}_{j} & =(n-1 n) \cap(j j+11), & I_{j} & =(j j+1) \cap(n-1 n 1), \\
\hat{n}_{A B} & =(n-1 n) \cap(A B 1), & \hat{A} & =(A B) \cap(n-1 n 1) .
\end{aligned}
$$

Explicit BCFW representation of the 2-loop amplitude is written in [13]. This BCFW representation has spurious poles in each term.

The another representation is the local representation. Each term of the local representation has no spurious pole. The local representation of the 2-loop MHV amplitudes is obtained from the sum of the double pentagon diagrams [12]. The double pentagon diagram is

$$
\begin{aligned}
& \times \frac{\langle A B(i-1 i i+1) \cap(j-1 j j+1)\rangle\langle i j k l\rangle}{\langle C D k-1 k\rangle\langle C D k k+1\rangle\langle C D l-1 l\rangle\langle C D l l+1\rangle} \\
& =Q_{i j k l} .
\end{aligned}
$$


This diagram has no spurious pole and the full amplitude is the cyclic sum over this diagram. For example, the local representation of the 2-loop MHV 4-point amplitude is

$$
\begin{aligned}
\mathcal{A}_{\mathrm{MHV}}^{2-\text { loop, } 4-\mathrm{pt}}= & Q_{1234}+Q_{2341}+Q_{3412}+Q_{4123} \\
= & \frac{\langle 1234\rangle^{3}}{\langle A B 12\rangle\langle A B 14\rangle\langle A B 23\rangle\langle A B C D\rangle\langle C D 14\rangle\langle C D 23\rangle\langle C D 34\rangle} \\
& +\frac{\langle 1234\rangle^{3}}{\langle A B 12\rangle\langle A B 14\rangle\langle A B 34\rangle\langle A B C D\rangle\langle C D 12\rangle\langle C D 23\rangle\langle C D 34\rangle} \\
& +\frac{\langle 1234\rangle^{3}}{\langle A B 12\rangle\langle A B 23\rangle\langle A B 34\rangle\langle A B C D\rangle\langle C D 12\rangle\langle C D 14\rangle\langle C D 34\rangle} \\
& +\frac{\langle 1234\rangle^{3}}{\langle A B 14\rangle\langle A B 23\rangle\langle A B 34\rangle\langle A B C D\rangle\langle C D 12\rangle\langle C D 14\rangle\langle C D 23\rangle}
\end{aligned}
$$

The 5-point case is

$$
\mathcal{A}_{\mathrm{MHV}}^{2-\text { loop, } 5-\mathrm{pt}}=(1,2,3,4)+(2,3,4,5)+(3,4,5,1)+(4,5,1,2)+(5,1,2,3)
$$

where $(i, j, k, l)=Q_{i j k l}+Q_{j k l i}+Q_{k l i j}+Q_{l i j k}$. The general $n$-point amplitude is

$$
\mathcal{A}_{\mathrm{MHV}}^{2-\text { loop }}=\sum_{i<j<k<l<i}
$$

where $i, j, k, l=1, \cdots, n$ and the notation $i<j<k<l<i$ in the sum is the cyclic sum.

In this representation there are no spurious poles and this local representation of the amplitudes is corresponding to the BCFW [12]. Because of this corresponding, it is enough to compare with the local representation. Next we construct the triangulation of the 2-loop MHV amplituhedron from the geometry using the sign flip definition and compare with the full form and the local representation from the double pentagon diagrams.

\section{Triangulation of 2-loop MHV amplituhedron from sign flips}

In this section, we see that the sign flip pattern gives a natural triangulation of the amplituhedron. First we consider the tree $m=1, k=1$ case. From the definition of sign flips, $\{\langle Y 1\rangle, \cdots,\langle Y n\rangle\}$ has 1 sign flip. We denote the place where the sign flip takes place $j$; $\langle Y j\rangle\left\langle 0\right.$ and $\langle Y j+1\rangle>0$. Now we can expand $Y$ on some basis $\mathcal{Z}_{A}, \mathcal{Z}_{B}$ as $Y=\mathcal{Z}_{A}+x \mathcal{Z}_{B}$. In order to describe the $m=1$ cell where the sign flip occurs at $j$, it is convenient to choose $\mathcal{Z}_{A}=\mathcal{Z}_{j}, \mathcal{Z}_{B}=\mathcal{Z}_{j+1}$. From the sign flip conditions, we must have $x_{j}>0$ and conversely, every $Y$ of this form with $x>0$ will belong to this cell. Then the canonical form for this sign flip pattern is

$$
\Omega_{j}=\frac{d x_{j}}{x_{j}}
$$


and the full form of $m=1 k=1$ amplituhedron is

$$
\Omega=\sum_{1 \leq j \leq n-1} \frac{d x_{j}}{x_{j}}
$$

This is the triangulation of the $m=1, k=1$ tree amplituhedron from sign flips. We can similarly triangulate for general $k$. The region in the $m=1, k$ amplituhedron where $\{\langle Y 1\rangle \cdots\langle Y n\rangle\}$ flips in slots $\left(j_{1}, \cdots j_{k}\right)$ is covered by

$$
Y=\left(\mathcal{Z}_{j_{1}}+x_{1} \mathcal{Z}_{j_{1}+1}\right)\left(\mathcal{Z}_{j_{2}}+x_{2} \mathcal{Z}_{j_{2}+1}\right) \cdots\left(\mathcal{Z}_{j_{k}}+x_{k} \mathcal{Z}_{j_{k}+1}\right) \text { with } x_{k}>0
$$

The $k$-form related to each cell is

$$
\Omega^{\left\{j_{1}, \cdots j_{k}\right\}}=\prod_{\alpha=1}^{k} d \log x_{\alpha}=\prod_{\alpha=1}^{k} d \log \frac{\left\langle Y \mathcal{Z}_{i_{\alpha}+1}\right\rangle}{\left\langle Y \mathcal{Z}_{i_{\alpha}}\right\rangle}
$$

and the full form is

$$
\Omega=\sum_{2 \leq j_{1} \leq j_{2} \leq \cdots \leq j_{k} \leq n-1} \Omega^{\left\{j_{1}, \cdots j_{k}\right\}} .
$$

The dimension of each cell is $k$ and this is the triangulation of $\mathcal{A}(m=1, k, n)$ into nonredundant cells. Similarly the $m=2$ amplituhedron can be triangulated from the sign flips. For $m=2$ case, the sequence $\{\langle Y 12\rangle,\langle Y 13\rangle, \cdots,\langle Y 1 n\rangle\}$ has $k$ sign flip in the slots $\left(j_{1}, \cdots j_{k}\right)$. Then we can expand $Y$ as

$Y=\left(+\mathcal{Z}_{1}+x_{1} \mathcal{Z}_{j_{1}}+y_{1} \mathcal{Z}_{1}+1\right)\left(-\mathcal{Z}_{1}+x_{2} \mathcal{Z}_{j_{2}}+y_{2} \mathcal{Z} j_{2}+1\right) \cdots\left((-1)^{k} \mathcal{Z}_{1}+x_{k} \mathcal{Z}_{j_{k}}+y_{k} \mathcal{Z}_{k}+1\right)$

with $x_{k}, y_{k}>0$. The $k$-form for each cell is

$$
\Omega^{\left\{j_{1}, \cdots j_{k}\right\}}=\prod_{\alpha=1}^{k} d \log x_{\alpha} d \log y_{\alpha}=\prod_{\alpha=1}^{k} d \log \frac{\left\langle Y 1 i_{\alpha}\right\rangle}{\left\langle Y i_{\alpha} i_{\alpha}+1\right\rangle} d \log \frac{\left\langle Y 1 i_{\alpha}+1\right\rangle}{\left\langle Y i_{\alpha} i_{\alpha}+1\right\rangle}
$$

and the full form is

$$
\Omega=\sum_{2 \leq j_{1} \leq j_{2} \leq \cdots \leq j_{k} \leq n-1} \Omega^{\left\{j_{1}, \cdots j_{k}\right\}} .
$$

The dimension of each cell is $2 k$ and this is the triangulation of $\mathcal{A}(m=2, k, n)$. The important case is $k=2$, it is isomorphic to the 1-loop MHV amplituhedron $\mathcal{A}(k=0$, $n, m=4, l=1)$. The full canonical form of this amplituhedron $\mathcal{A}(2, n, 2)$ from sign flips is

$$
\begin{aligned}
\Omega= & \sum_{2 \leq j_{1} \leq j_{2} \leq \cdots \leq j_{k} \leq n-1} \Omega^{\left\{j_{1}, \cdots j_{k}\right\}} \\
= & \left\langle Y d^{2} Y_{1}\right\rangle\left\langle Y d^{2} Y_{2}\right\rangle \\
& \times \sum_{2 \leq j_{1} \leq j_{2} \leq \cdots \leq j_{k} \leq n-1} \frac{\left\langle Y_{1} Y_{2}(1 i i+1) \cap(1 j j+1)\right\rangle^{2}}{\langle Y 1 i\rangle\langle Y 1 i+1\rangle\langle Y i i+1\rangle\langle Y 1 j\rangle\langle Y 1 j+1\rangle\langle Y j j+1\rangle} .
\end{aligned}
$$

Take $\left(Y_{1}, Y_{2}\right) \leftrightarrow(A, B)$, this form corresponds to the Kermit representation of 1-loop MHV amplituhedron, we have already known that this is the triangulation of this amplituhedron. 
For $m=1$ and $m=2$ case, we have seen that the sign flip pattern gives us a triangulation of the amplituhedron and it comes from the geometry directly. However, for the case of $m=4$, there isn't a simple relation between the triangulation and the sign flip pattern. The case of the 1-loop MHV amplituhedron is an exception due to the isomorphism. However, we will see that we can triangulate the 2-loop MHV amplituhedron $\mathcal{A}(k=0, n, l=2)$ using the sign flip definition. The sign flip definition of the 2-loop MHV amplituhedron is

$$
\begin{aligned}
& \langle A B i i+1\rangle>0,\langle C D i i+1\rangle>0 \\
& \{\langle A B 12\rangle,\langle A B 13\rangle, \cdots,\langle A B 1 n\rangle\} \text { has } 2 \text { sign flip } \\
& \{\langle C D 12\rangle,\langle C D 13\rangle, \cdots,\langle C D 1 n\rangle\} \text { has } 2 \text { sign flip } \\
& \langle A B C D\rangle>0,
\end{aligned}
$$

$(A, B)$ and $(C, D)$ are the loop momentum for each amplituhedron. From this, we can see that the 2-loop MHV amplituhedron is constructed by two 1-loop MHV amplituhedron $(A B),(C D)$ and a further constraint $\langle A B C D\rangle>0$. The important fact is that even if we consider the general $n$-point, there is only one constraint $\langle A B C D\rangle>0$. Because of this, to obtain the canonical form we need to solve only one constraint and it is very easy rather than the $Y=C \cdot Z$ description. In the next section, we construct the triangulation of the 2-loop MHV amplituhedron from the sign flip definition and compare with the BCFW and the double pentagon representation.

\subsection{Four and five point amplitudes}

First we consider the simplest case, 2-loop 4-point MHV amplituhedron. From the sign flip definition (1.6), it is constructed from the two 1-loop 4-point MHV. The sign flip definition of the 1-loop amplituhedron is

$$
\begin{aligned}
& \langle A B i i+1\rangle>0, \\
& \{\langle A B 12\rangle,\langle A B 13\rangle,\langle A B 14\rangle\} \text { has } 1 \text { sign flip. }
\end{aligned}
$$

From this, there is only 1 sign flip pattern

$$
\{\langle A B 12\rangle,\langle A B 13\rangle,\langle A B 14\rangle\}=\{+,-,+\} .
$$

Then we can expand the loop momentum as

$$
Z_{A}=Z_{1}+x_{1} Z_{2}+w_{1} Z_{3}, \quad Z_{B}=-Z_{1}+y_{1} Z_{3}+z_{1} Z_{4}
$$

From the sign flip condition, the region of these variables are $x_{1}, w_{1}, y_{1}, z_{1}>0$. In the view of the $Y_{\alpha}=C_{\alpha a} \mathcal{Z}_{a}$ description, the $C$-matrix of this sign flip pattern is

$$
C=\left(\begin{array}{cccc}
1 & x_{1} & w_{1} & 0 \\
-1 & 0 & y_{1} & z_{1}
\end{array}\right) \text {. }
$$

Boundary of this pattern is $x_{1} \rightarrow 0, w_{1} \rightarrow 0, y_{1} \rightarrow 0, z_{1} \rightarrow 0$, then the canonical form is

$$
\Omega_{4}^{l=1}=\frac{d x_{1}}{x_{1}} \frac{d w_{1}}{w_{1}} \frac{d y_{1}}{y_{1}} \frac{d z_{1}}{z_{1}}=\frac{\left\langle A B d^{2} A\right\rangle\left\langle A B d^{2} B\right\rangle\langle 1234\rangle^{2}}{\langle A B 12\rangle\langle A B 23\rangle\langle A B 34\rangle\langle A B 14\rangle} .
$$


This form corresponds to the form of the 4-point 1-loop MHV amplituhedron obtained from the $Y=C \cdot Z$ description $[5,6]$. Next we consider the 2-loop 4-point MHV amplituhedron. This is constructed from the two 1-loop amplituhedron and a constraint $\langle A B C D\rangle>0$. We can parametrize these two 1-loop amplituhedron as

$$
\begin{aligned}
& Z_{A}=Z_{1}+x_{1} Z_{2}+w_{1} Z_{3}, \quad Z_{B}=-Z_{1}+y_{1} Z_{3}+z_{1} Z_{4} \\
& Z_{C}=Z_{1}+x_{2} Z_{2}+w_{2} Z_{3}, \quad Z_{D}=-Z_{1}+y_{2} Z_{3}+z_{2} Z_{4} \\
& \text { with } \quad x_{1}, w_{1}, y_{1}, z_{1}, x_{2}, w_{2}, y_{2}, z_{2}>0 .
\end{aligned}
$$

In view of the $\mathcal{Y}=\mathcal{C} \cdot Z$ description, the $C$-matrix is

$$
C=\left(\begin{array}{cccc}
1 & x_{1} & w_{1} & 0 \\
-1 & 0 & y_{1} & z_{1} \\
1 & x_{2} & w_{2} & 0 \\
-1 & 0 & y_{2} & z_{2}
\end{array}\right)
$$

Under this parametrization, the constraint become

$$
\langle A B C D\rangle=\langle 1234\rangle\left\{\left(x_{1}-x_{2}\right)\left(y_{1} z_{2}-y_{2} z_{1}\right)+\left(z_{1}-z_{2}\right)\left(w_{1} x_{2}-w_{2} x_{1}\right)\right\}>0 .
$$

From this condition, these parameters are bounded further. Without loss of generality, we can take $y_{1} z_{2}-y_{2} z_{1}>0$. Then from $\langle A B C D\rangle>0$,

$$
x_{1}>x_{2}-\frac{\left(z_{1}-z_{2}\right)\left(w_{1} x_{2}-w_{2} x_{1}\right)}{y_{1} z_{2}-y_{2} z_{1}}=x_{2}-a .
$$

Therefore there are 4 cases depending on the signs of $\left(z_{1}-z_{2}\right),\left(w_{1} x_{2}-w_{2} x_{1}\right)$. For example, the case of $\left(z_{1}-z_{2}\right)>0,\left(w_{1} x_{2}-w_{2} x_{1}\right)>0$, the regions of these variables are

$$
\begin{aligned}
& x_{2}+a>x_{1}>0, \quad w_{1}>\frac{x_{1}}{x_{2}} w_{2}, \quad y_{1}>\frac{z_{1}}{z_{2}} y_{2}, \quad z_{1}>z_{2} \\
& x_{2}>0, \quad w_{2}>0, \quad y_{2}>0, \quad z_{2}>0 .
\end{aligned}
$$

Compare with (3.16), the regions of these parameters are further bounded because of this constraint. Then there are 9 boundaries

$$
\begin{array}{rlrlrl}
\left(x_{1} \rightarrow x_{2}+a,\right. & \left.x_{1} \rightarrow 0\right), & w_{1} \rightarrow \frac{x_{1}}{x_{2}} w_{2}, & y_{1} \rightarrow \frac{z_{1}}{z_{2}} y_{2}, & z_{1} \rightarrow z_{2} \\
x_{2} \rightarrow 0, & w_{2} \rightarrow 0, & y_{2} \rightarrow 0, & z_{2} \rightarrow 0 .
\end{array}
$$

We can obtain the canonical form for this case. For example, the region of $x_{1}$ is $0<x_{1}<$ $x_{2}+a$, then the form for $x_{1}$ is

$$
\frac{1}{x_{1}}-\frac{1}{x_{1}-x_{2}-a}
$$

Then the canonical form for this case is

$$
\Omega=\frac{1}{x_{2}}\left(\frac{1}{x_{1}}-\frac{1}{x_{1}-x_{2}-a}\right) \frac{1}{w_{1}-\frac{x_{1}}{x_{2}} w_{2}} \frac{1}{w_{2}} \frac{1}{y_{1}-\frac{z_{1}}{z_{2}} y_{2}} \frac{1}{y_{2}} \frac{1}{z_{1}-z_{2}} \frac{1}{z_{2}} .
$$


There are 4 patterns depending on the signs of $\left(z_{1}-z_{2}\right),\left(w_{1} x_{2}-w_{2} x_{1}\right)$. The forms related to these 4 patterns can be constructed similarly

$$
\begin{aligned}
& \Omega_{1}=\frac{1}{x_{1}-x_{2}+a} \frac{1}{x_{2}}\left(\frac{1}{w_{1}}-\frac{1}{w_{1}-\frac{x_{1}}{x_{2}} w_{2}}\right) \frac{1}{w_{2}} \frac{1}{y_{1}-\frac{z_{1}}{z_{2}} y_{2}} \frac{1}{y_{2}} \frac{1}{z_{1}-z_{2}} \frac{1}{z_{2}} \\
& \Omega_{2}=\frac{1}{x_{1}-x_{2}+a} \frac{1}{x_{2}} \frac{1}{w_{1}-\frac{x_{1}}{x_{2}} w_{2}} \frac{1}{w_{2}} \frac{1}{y_{1}-\frac{z_{1}}{z_{2}} y_{2}} \frac{1}{y_{2}}\left(\frac{1}{z_{1}}-\frac{1}{z_{1}-z_{2}}\right) \frac{1}{z_{2}} \\
& \Omega_{3}=\frac{1}{x_{1}}\left(\frac{1}{x_{2}}-\frac{1}{x_{2}-x_{1}-a}\right) \frac{1}{w_{1}-\frac{x_{1}}{x_{2}} w_{2}} \frac{1}{w_{2}} \frac{1}{y_{1}-\frac{z_{1}}{z_{2}} y_{2}} \frac{1}{y_{2}} \frac{1}{z_{1}-z_{2}} \frac{1}{z_{2}} \\
& \Omega_{4}=\frac{1}{x_{1}}\left(\frac{1}{x_{2}}-\frac{1}{x_{2}-x_{1}-a}\right)\left(\frac{1}{w_{1}}-\frac{1}{w_{1}-\frac{x_{1}}{x_{2}} w_{2}}\right) \frac{1}{w_{2}} \frac{1}{y_{1}-\frac{z_{1}}{z_{2}} y_{2}} \frac{1}{y_{2}}\left(\frac{1}{z_{1}}-\frac{1}{z_{1}-z_{2}}\right) \frac{1}{z_{2}} .
\end{aligned}
$$

The remaining four cases $y_{1} z_{2}-y_{2} z_{1}<0$ are obtained that swap $1 \leftrightarrow 2$. The sum of these 8 form is

$$
\Omega_{4 \mathrm{pt}}^{l=2}=\frac{d x_{1} d x_{2} d w_{1} d w_{2} d y_{1} d y_{2} d z_{1} d z_{2}}{x_{1} x_{2} w_{1} w_{2} y_{1} y_{2} z_{1} z_{2}} \frac{\left(x_{1} y_{1} z_{2}+x_{2} y_{2} z_{1}+x_{2} w_{1} z_{1}+x_{1} w_{2} z_{2}\right)}{\left\{\left(x_{1}-x_{2}\right)\left(y_{1} z_{2}-y_{2} z_{1}\right)+\left(z_{1}-z_{2}\right)\left(w_{1} x_{2}-w_{2} x_{1}\right)\right\}} .
$$

To translate it into the momentum twistor, we need to solve (3.16) for $x_{1}, x_{2}, \cdots, z_{2}$

$$
\begin{array}{llll}
x_{1}=-\frac{\langle A B 13\rangle}{\langle A B 23\rangle}, & w_{1}=\frac{\langle A B 12\rangle}{\langle A B 23\rangle}, & y_{1}=\frac{\langle A B 14\rangle}{\langle A B 34\rangle}, & z_{1}=-\frac{\langle A B 13\rangle}{\langle A B 34\rangle} \\
x_{2}=-\frac{\langle C D 13\rangle}{\langle C D 23\rangle}, & w_{2}=\frac{\langle C D 12\rangle}{\langle C D 23\rangle}, & y_{2}=\frac{\langle C D 14\rangle}{\langle C D 34\rangle}, & z_{2}=-\frac{\langle C D 13\rangle}{\langle C D 34\rangle} .
\end{array}
$$

Then the full form in the momentum twistor space is

$$
\begin{aligned}
\Omega_{4 \mathrm{pt}}^{l=2}= & \frac{\langle 1234\rangle^{3}\left\langle A B d^{2} A\right\rangle\left\langle A B d^{2} B\right\rangle\left\langle C D d^{2} C\right\rangle\left\langle C D d^{2} D\right\rangle}{\langle A B 12\rangle\langle A B 14\rangle\langle A B 23\rangle\langle A B 34\rangle\langle A B C D\rangle\langle C D 12\rangle\langle C D 14\rangle\langle C D 23\rangle\langle C D 34\rangle} \\
& \times\{\langle A B 34\rangle\langle C D 12\rangle+\langle A B 23\rangle\langle C D 14\rangle+\langle A B 14\rangle\langle C D 23\rangle+\langle A B 12\rangle\langle C D 34\rangle\} .
\end{aligned}
$$

This result is corresponding to the local representation of the 4-point amplitude (2.4). The dimension of this amplituhedron is 8, therefore in this 4-point case, it is just a nonredundant cell. Of cause it can be obtained from the $Y=C \cdot Z$ description directly [6] and our result is corresponding to this $Y=C \cdot Z$ result. Next we see that the higher point 2-loop MHV amplituhedron can be triangulated into the non-redundant dimension 8 cells.

Next we consider the 5-point amplitude. The 2-loop 5-point MHV amplituhedron is constructed from the two 1-loop 5-point MHV amplituhedron and a further constraint. In the 1-loop $n=5, k=2$ amplitude, there are 3 patterns of sign flips as

$$
\{\langle A B 12\rangle,\langle A B 13\rangle,\langle A B 14\rangle,\langle A B 15\rangle\}=\{+,-,+,+\} \text { or }\{+,-,-,+\} \text { or }\{+,+,-,+\} \text {. }
$$


Then we can parametrize for each pattern as

$$
\begin{aligned}
& \left\{\begin{array} { l } 
{ Z _ { A } = Z _ { 1 } + x _ { 1 } Z _ { 2 } + w _ { 1 } Z _ { 3 } } \\
{ Z _ { B } = - Z _ { 1 } + y _ { 1 } Z _ { 3 } + z _ { 1 } Z _ { 4 } }
\end{array} \quad ( 2 , 3 ) \text { pattern, } \left\{\begin{array}{l}
Z_{A}=Z_{1}+x_{1} Z_{2}+w_{1} Z_{3} \\
Z_{B}=-Z_{1}+y_{1} Z_{4}+z_{1} Z_{5}
\end{array} \quad(2,4)\right.\right. \text { pattern, } \\
& \left\{\begin{array}{l}
Z_{A}=Z_{1}+x_{1} Z_{3}+w_{1} Z_{4} \\
Z_{B}=-Z_{1}+y_{1} Z_{4}+z_{1} Z_{5}
\end{array} \quad(3,4)\right. \text { pattern. }
\end{aligned}
$$

Then depending on which pattern (3.29) we choose, there are $3 \times 3=9$ patterns in the 2 -loop amplituhedron. We can expect that the full form of the 2-loop 5-point MHV amplituhedron is obtained by the sum of these forms related to each 9 pattern. Each form can be obtained similarly as the 4-pt case, and the explicit calculation is given in the appendix and here we will write only the results. The case of $(2,3) \times(2,3)$ is same as the 4 -pt case. The case of $(3,4) \times(3,4)$,

$$
\begin{aligned}
\Omega_{3434}= & \frac{d x_{1} d x_{2} d w_{1} d w_{2} d y_{1} d y_{2} d z_{1} d z_{2}}{x_{1} x_{2} w_{1} w_{2} y_{1} y_{2} z_{1} z_{2}} \frac{\langle 1345\rangle}{\langle A B C D\rangle}\left(x_{1} y_{1} z_{2}+x_{2} y_{2} z_{1}+x_{2} w_{1} z_{1}+x_{1} w_{2} z_{2}\right) \\
= & \frac{\langle 1345\rangle^{3}\left\langle A B d^{2} A\right\rangle\left\langle A B d^{2} B\right\rangle\left\langle C D d^{2} C\right\rangle\left\langle C D d^{2} D\right\rangle}{\langle A B 13\rangle\langle A B 15\rangle\langle A B 34\rangle\langle A B 45\rangle\langle A B C D\rangle\langle C D 13\rangle\langle C D 15\rangle\langle C D 34\rangle\langle C D 45\rangle} \\
& \times\{\langle A B 45\rangle\langle C D 13\rangle+\langle A B 34\rangle\langle C D 15\rangle+\langle A B 15\rangle\langle C D 34\rangle+\langle A B 13\rangle\langle C D 45\rangle\} .
\end{aligned}
$$

The case of $(2,4) \times(3,4)$,

$$
\begin{aligned}
\Omega_{2434}= & \frac{\left\langle 123 A_{4}\right\rangle\left\langle 134 C_{4}\right\rangle\left\langle A B d^{2} A\right\rangle\left\langle A B d^{2} B\right\rangle\left\langle C D d^{2} C\right\rangle\left\langle C D d^{2} D\right\rangle}{\left\{\begin{array}{c}
\langle A B 12\rangle\langle A B 13\rangle\langle A B 14\rangle\langle A B 15\rangle\langle A B 23\rangle\langle A B 45\rangle \\
\times\langle A B C D\rangle\langle C D 13\rangle\langle C D 14\rangle^{2}\langle C D 15\rangle\langle C D 34\rangle\langle C D 45\rangle
\end{array}\right\}} \\
& \times\left\{\left\langle 123 A_{4}\right\rangle(\langle A B 45\rangle\langle C D 13\rangle\langle C D 14\rangle+\langle A B 15\rangle\langle C D 34\rangle\langle C D 14\rangle)\right. \\
& \left.-\left\langle 345 A_{2}\right\rangle\langle A B 14\rangle\langle C D 14\rangle\langle C D 15\rangle+\left\langle 123 C_{4}\right\rangle\langle C D 14\rangle\langle A B 45\rangle\langle A B 13\rangle\right\} .
\end{aligned}
$$

The case of $(2,3) \times(3,4)$,

$$
\begin{aligned}
\Omega_{2334}= & \frac{\left\langle 123 A_{3}\right\rangle\left\langle 134 C_{4}\right\rangle\left\langle A B d^{2} A\right\rangle\left\langle A B d^{2} B\right\rangle\left\langle C D d^{2} C\right\rangle\left\langle C D d^{2} D\right\rangle}{\langle A B 12\rangle\langle A B 13\rangle^{2}\langle A B 14\rangle\langle A B 23\rangle\langle A B 34\rangle} \\
& \left\{\begin{array}{c}
\times A B C D\rangle\langle C D 13\rangle\langle C D 14\rangle^{2}\langle C D 15\rangle\langle C D 34\rangle\langle C D 45\rangle
\end{array}\right\} \\
& \times\left\{\langle A B 13\rangle\left\langle 123 C_{4}\right\rangle\left\langle C D 4 A_{3}\right\rangle-\langle A B 13\rangle\langle A B 14\rangle\langle C D 13\rangle\left\langle 234 C_{4}\right\rangle\right. \\
& \left.+\langle C D 14\rangle\left\langle 145 A_{2}\right\rangle\left\langle C D 3 A_{3}\right\rangle-\langle A B 14\rangle\langle A B 23\rangle\langle C D 13\rangle\langle C D 14\rangle\langle 1345\rangle\right\} .
\end{aligned}
$$


The case of $(2,4) \times(2,4)$,

$$
\begin{aligned}
& \Omega_{2424}=\frac{\left\langle 123 A_{4}\right\rangle\left\langle 123 C_{4}\right\rangle\left\langle A B d^{2} A\right\rangle\left\langle A B d^{2} B\right\rangle\left\langle C D d^{2} C\right\rangle\left\langle C D d^{2} D\right\rangle}{\left\{\begin{array}{c}
\langle A B 12\rangle\langle A B 13\rangle\langle A B 14\rangle\langle A B 15\rangle\langle A B 23\rangle\langle A B 45\rangle\langle A B C D\rangle \\
\times\langle C D 12\rangle\langle C D 13\rangle\langle C D 14\rangle\langle C D 15\rangle\langle C D 23\rangle\langle C D 45\rangle
\end{array}\right\}} \\
& \times\left\{\left\langle 123 A_{4}\right\rangle(\langle A B 12\rangle\langle C D 13\rangle\langle C D 45\rangle+\langle A B 15\rangle\langle C D 14\rangle\langle C D 23\rangle)\right. \\
& +\left\langle 123 C_{4}\right\rangle(\langle A B 13\rangle\langle A B 45\rangle\langle C D 12\rangle+\langle A B 14\rangle\langle A B 23\rangle\langle C D 15\rangle) \\
& +\langle 2345\rangle(\langle A B 12\rangle\langle A B 15\rangle\langle C D 13\rangle\langle C D 14\rangle+\langle A B 13\rangle\langle A B 14\rangle\langle C D 12\rangle\langle C D 15\rangle)\} .
\end{aligned}
$$

We use the symbols that

$$
A_{i} \equiv(A B) \cap(1 i i+1), \quad C_{k} \equiv(C D) \cap(1 k k+1) .
$$

The remaining patterns are $(3,4) \times(2,3),(2,4) \times(2,3),(3,4) \times(2,4)$. These forms can be obtained from $\Omega_{2334}, \Omega_{2324}, \Omega_{2434}$ that swap $A B \leftrightarrow C D$. We obtain all 9 forms and we can calculate the sum of these forms

$$
\Omega_{5 \text {-pt }}^{l=2, \mathrm{MHV}}=\Omega_{2323}+\Omega_{2424}+\Omega_{3434}+\Omega_{2324}+\Omega_{2334}+\Omega_{2434}+\Omega_{2423}+\Omega_{3423}+\Omega_{3424} .
$$

Each form $\Omega_{i j k l}$ has spurious poles $\langle A B 13\rangle,\langle A B 14\rangle,\langle C D 13\rangle,\langle C D 14\rangle$, we can see that all of these are canceled and remain only the physical poles in the full form. This result is corresponding to the local representation (2.5). From this result, we can see that the 2loop 5-point MHV amplituhedron is triangulated into the 9 cells related to each sign flip pattern, and these cells are 8-dimensional cells $G_{+}(4,4)$.

In the case of the BCFW, each cell of the 2-loop 5-point MHV amplitude has also the spurious poles not only like $\langle A B 13\rangle,\langle A B 14\rangle,\langle C D 13\rangle,\langle C D 14\rangle$, but also more complicate poles from taking the forward limit. Therefore this triangulation has a different structure compared with the BCFW triangulation.

\section{$3.2 \quad$ n-point amplitude}

Next we consider the general n-pt case. First we consider the 1-loop n-point MHV amplituhedron. There are $\frac{1}{2}(n-3)(n-2)$ sign flip patterns from the way to chose $i, j$ that

$$
i, j=2,3, \cdots, n-1, \quad i<j .
$$

When sign flip occurs at $i, j$ slots, we can parametrize the loop momentum as

$$
Z_{A}=Z_{1}+x Z_{i}+w Z_{i+1}, Z_{B}=-Z_{1}+y Z_{j}+z Z_{j+1},
$$

and the canonical form of this pattern is

$$
\Omega_{i j}=\frac{d x}{x} \frac{d w}{w} \frac{d y}{y} \frac{d z}{z}
$$

Then the full form is

$$
\Omega=\sum_{\substack{i, j=2,3, \cdots, n-1 \\ i<j}} \Omega_{i j}
$$


Next we consider the 2-loop $n$-point MHV amplituhedron. There are $\left[\frac{1}{2}(n-3)(n-2)\right]^{2}$ sign flip patterns in the 2-loop n-point MHV amplituhedron depending on the way to chose $i, j, k, l$ that

$$
i, j, k, l=2,3, \cdots, n-1, \quad i<j, k<l \text {. }
$$

We can expand as

$$
\left\{\begin{array} { l } 
{ Z _ { A } = Z _ { 1 } + x _ { 1 } Z _ { i } + w _ { 1 } Z _ { i + 1 } , } \\
{ Z _ { B } = - Z _ { 1 } + y _ { 1 } Z _ { j } + z _ { 1 } Z _ { j + 1 } }
\end{array} \quad \left\{\begin{array}{l}
Z_{C}=Z_{1}+x_{2} Z_{k}+w_{2} Z_{k+1} \\
Z_{D}=-Z_{1}+y_{2} Z_{l}+z_{2} Z_{l+1}
\end{array} .\right.\right.
$$

From the constraint $\langle A B C D\rangle>0$, these parameters are bounded. The region of these parameters are depending on the other parameters and $\langle i j k l\rangle$, however, the sign of this determinant changes depending on the relation between $(i, j)$ and $(k, l)$. More precisely, the sign is depending on the order of $i, j, k, l$, if $i<j<k<l$, then $\langle i j k l\rangle>0$. Therefore we need to determine the order of $i, j, k, l$ to calculate each form. This order of $i, j, k, l$ can be divided into 13 groups as

$$
\begin{aligned}
& i<k<l<j \cdots(1), \quad i<k<j<l \cdots(2), \quad i<j<k<l \cdots(3), \quad i=k<l<j \cdots(4), \\
& i=k<j=l \cdots(5), \quad i=k<j<l \cdots(6), \quad i<k<j=l \cdots(7), \quad i<j=k<l \cdots(8), \\
& k<i=l<j \cdots(9), \quad k<i<l<j \cdots(10), \quad k<i<j=l \cdots(11), \quad k<i<j<l \cdots(12), \\
& k<l<i<j \cdots(13) \text {. }
\end{aligned}
$$

We can compute the forms for each case in the same way as the 5-point case. The case of (1), $C$-matrix is

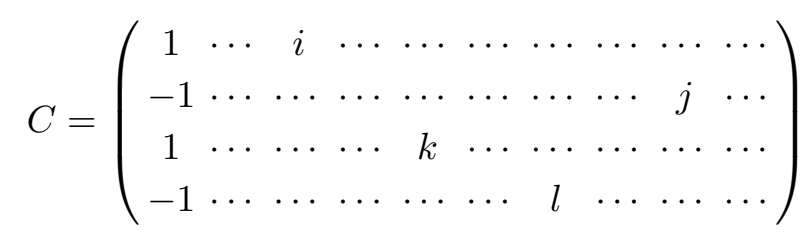

where

$$
\left(i, i+1 \rightarrow x_{1}, w_{1}\right) \quad\left(j, j+1 \rightarrow y_{1}, z_{1}\right) \quad\left(k, k+1 \rightarrow x_{2}, w_{2}\right) \quad\left(l, l+1 \rightarrow y_{2}, z_{2}\right), \cdots=0 .
$$

The canonical form of this case in the momentum twistor space is

$$
\Omega_{i j k l}^{1}=\frac{\omega_{i j k l}^{1^{\prime}}\left\langle 1 i i+1 A_{j}\right\rangle\left\langle 1 k k+1 C_{l}\right\rangle\left\langle A B d^{2} A\right\rangle\left\langle A B d^{2} B\right\rangle\left\langle C D d^{2} C\right\rangle\left\langle C D d^{2} D\right\rangle}{\langle A B 1 i\rangle\langle A B 1 i+1\rangle\langle A B 1 j\rangle\langle A B 1 j+1\rangle\langle A B C D\rangle\langle C D 1 k\rangle\langle C D 1 k+1\rangle\langle C D 1 l\rangle\langle C D 1 l+1\rangle}
$$

where

$$
\omega_{i j k l}^{1^{\prime}}=\frac{\langle A B i i+1\rangle\left\langle A_{j} C_{k} C_{l} 1\right\rangle+\left\langle A_{i} A_{j} C_{k} C_{l}\right\rangle}{\langle A B i i+1\rangle\langle A B j j+1\rangle\langle C D k k+1\rangle\langle C D l l+1\rangle} .
$$

Again we use the symbols (3.34). The canonical forms for another case can be obtained similarly. We give all the canonical forms and the explicit calculation of the case of (1) in the appendix.

Then the full form of the 2-loop n-pt MHV amplituhedron is

$$
\Omega_{\mathrm{MHV}}^{n \text {-pt 2-loop }}=\sum_{\substack{i, j, k, l=2,3, \cdots, n-1 \\ i<k<l<j}} \Omega_{i j k l}^{1}+\sum_{i<k<j<l} \Omega_{i j k l}^{2}+\sum_{i<j<k<l} \Omega_{i j k l}^{3}+\cdots+\sum_{k<l<i<j} \Omega_{i j k l}^{13} .
$$


Similarly for the 5-pt case, these cells have spurious poles. However, all of these poles are canceled and remain only physical poles. We compared this result and local representation (2.6) (or BCFW) numerically [13] and we checked that these results are corresponding up to at least 22-pt. From this results, we can see that the 2-loop n-pt MHV amplituhedron is triangulated into the $\left[\frac{1}{2}(n-3)(n-2)\right]^{2} 8$-dimension cells and this triangulation is obtained directly from the geometry.

\section{More 2-loop objects}

\subsection{Log of the 2-loop MHV amplitude}

In this section we consider the log of the 2-loop MHV amplitude. The expansion of the amplitude is

$$
\mathcal{A}=1+g A_{1}+g^{2} A_{2}+g^{3} A_{3}+\cdots .
$$

Then the expansion of the logarithm of the amplitude is

$$
\mathcal{S}=\log \mathcal{A}=g S_{1}+g^{2} S_{2}+g^{3} S_{3}+\cdots
$$

where $S_{L}$ is a sum of $A_{L}$ and products of lower-loop amplitude,

$$
S_{1}=A_{1}, \quad S_{2}=A_{2}-\frac{1}{2} A_{1}^{2}, \quad S_{3}=A_{3}-A_{2} A_{1}+\frac{1}{3} A_{1}^{3}, \quad \cdots,
$$

therefore the first non-trivial part is the 2-loop log amplitude. The 2-loop log amplitude can be expressed simply as a non-planar cyclic sum of the double pentagon diagram because of the simple relation between the square of the 1-loop pentagon diagram and the 2-loop double pentagon diagram [12]. The 1-loop pentagon diagram is

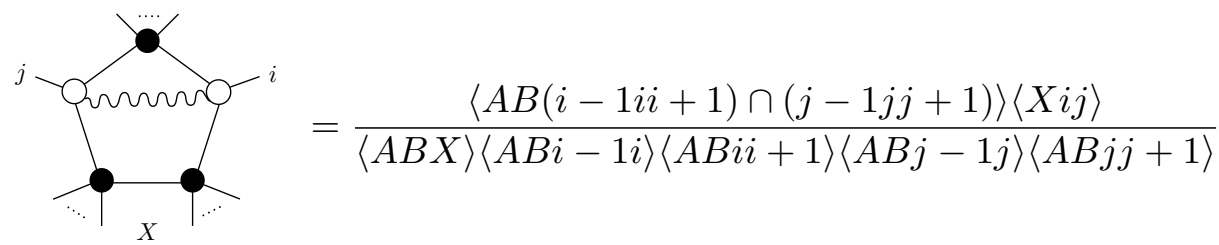

and the 1-loop MHV amplitude is

$$
\mathcal{A}_{\mathrm{MHV}}^{1-\text { loop }}=\sum_{i<j}\left\{\text { Cono }^{i}\right.
$$

The relation between this pentagon diagram and the double pentagon diagram is

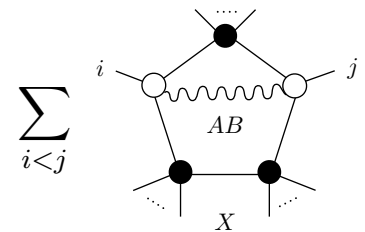

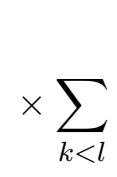

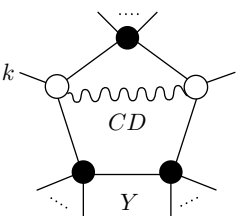

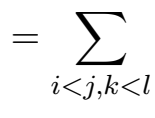

$=\sum_{i<j, k<l}$

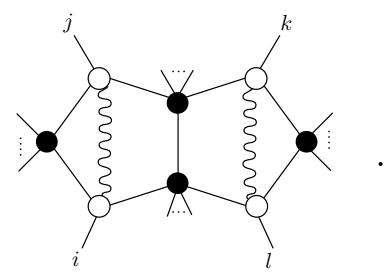


The left side is just $\left(\mathcal{A}_{\mathrm{MHV}}^{1-\text { loop }}\right)^{2}$ and the right side contains not only the planar diagrams $i<j<k<l$ but also the non-planar diagrams; for example, $i<k<j<l$. From (4.3), the log of the 2-loop amplitude is

$$
-[\log \mathcal{A}]_{\mathrm{MHV}}^{2-\text { loop }}=\frac{1}{2}\left(\mathcal{A}_{\mathrm{MHV}}^{1-\text { loop }}\right)^{2}-\mathcal{A}_{\mathrm{MHV}}^{2-\text { loop }} .
$$

This means that the sum of all non-planar double pentagon diagrams times minus sign gives us the log of the 2-loop amplitude [12]

$$
[\log \mathcal{A}]_{\mathrm{MHV}}^{2-\text { loop }}=-\sum_{i<k<j<l<i}
$$

For example, the log of the 4-pt amplitude is

$$
\begin{aligned}
{[\log \mathcal{A}]_{\mathrm{MHV}}^{2-\text { loop, }}{ }^{4-\mathrm{pt}} } & =-Q_{1324} \\
& =\frac{\langle 1234\rangle^{3}(\langle A B 13\rangle\langle C D 24\rangle+\langle A B 24\rangle\langle C D 13\rangle)}{\langle A B 12\rangle\langle A B 23\rangle\langle A B 34\rangle\langle A B 14\rangle\langle A B C D\rangle\langle C D 12\rangle\langle C D 23\rangle\langle C D 34\rangle\langle C D 14\rangle} .
\end{aligned}
$$

Next we consider the log of the amplitude from the geometrical view. First we consider the region of the log of the amplitude. In the case of the 2-loop MHV, the definition of the amplituhedron is

$$
\mathcal{L}_{i}=D_{i} \cdot Z, \quad i=1,2
$$

and

$$
\left\langle D_{(1)} D_{(2)}\right\rangle>0 \text {. }
$$

We consider another case: the square of the 1-loop MHV amplituhedron. This is defined similarly

$$
\mathcal{L}_{i}=D_{i} \cdot Z, \quad i=1,2 .
$$

However, this has no positivity condition. From (4.7), the region of the minus log of the amplitude is $\left\langle D_{(1)} D_{(2)}\right\rangle<0$. This pattern can be extended to all higher loop [6]. Then the question is that is it possible to obtain the log of the 2-loop MHV amplitude from the geometry

$$
\mathcal{L}_{i}=D_{i} \cdot Z, \quad i=1,2, \quad \text { and } \quad\left\langle D_{(1)} D_{(2)}\right\rangle<0
$$

and the canonical form of this geometry? In this section we construct the canonical form of this space (4.13) and see that it is corresponding to the log of the 2-loop MHV amplitude. To obtain the canonical form, we use the sign flip definition of this geometry

$$
\begin{aligned}
& \langle A B i i+1\rangle>0,\langle C D i i+1\rangle>0 \\
& \{\langle A B 12\rangle,\langle A B 13\rangle, \cdots,\langle A B 1 n\rangle\} \text { has } 2 \text { sign flip } \\
& \{\langle C D 12\rangle,\langle C D 13\rangle, \cdots,\langle C D 1 n\rangle\} \text { has } 2 \text { sign flip } \\
& \langle A B C D\rangle<0
\end{aligned}
$$


and call this geometry "2-loop MHV log amplituhedron". From this definition, we can see that the 2-loop n-point MHV log amplituhedron is constructed from the two 1-loop MHV amplituhedron and a negative constraint $\langle A B C D\rangle<0$. Then there are $\left[\frac{1}{2}(n-3)(n-2)\right]^{2}$ sign flip patterns in the 2-loop n-point MHV log amplituhedron depending on the way to chose $i, j, k, l$ that

$$
i, j, k, l=2,3, \cdots, n-1, \quad i<j, k<l .
$$

We can expand the loop momentum $\left(Z_{A}, Z_{B}\right),\left(Z_{C}, Z_{D}\right)$ as (3.6) and the order of $i, j, k, l$ is divided into 13 groups as (3.41). Once we get the order of $i, j, k, l$, then we can calculate the canonical form similarly. For example, the canonical form for the case of (1) is

$$
\Omega_{i j k l}^{1}[\log ]=\frac{d x_{1} d x_{2} \cdots d z_{1} d z_{2}}{x_{1} x_{2} w_{1} w_{2} y_{1} y_{2} z_{1} z_{2}} \frac{-1}{\left(a z_{2}-b w_{1}-c x_{1}-d w_{2}+e y_{2}\right)} \times \omega_{i j k l}^{1}[\log ]
$$

where

$$
\begin{aligned}
\omega_{i j k l}^{1}[\log ]= & x_{2} y_{1}\langle 1 i+1 k j\rangle+x_{2} z_{1}\langle 1 i+1 k j+1\rangle+y_{1} y_{2}\langle 1 i+1 l j\rangle+y_{2} z_{1}\langle 1 i+1 l j+1\rangle \\
& +y_{1} z_{2}\langle 1 i+1 l+1 j\rangle+z_{1} z_{2}\langle 1 i+1 l+1 j+1\rangle+x_{2} y_{1}\langle 1 i k j\rangle+x_{2} z_{1}\langle 1 i k j+1\rangle \\
& +w_{2} y_{1}\langle 1 i k+1 j\rangle+w_{2} z_{1}\langle 1 i k+1 j+1\rangle+y_{1} y_{2}\langle 1 i l j\rangle+y_{2} z_{1}\langle 1 i l+1 j\rangle \\
& +y_{1} z_{2}\langle 1 i l+1 j\rangle+z_{1} z_{2}\langle 1 i l+1 j+1\rangle+w_{1} y_{1}\langle 1 i+1 k+1 j\rangle \\
& +w_{1} z_{1}\langle 1 i+1 k+1 j+1\rangle
\end{aligned}
$$

and

$$
\begin{aligned}
a= & x_{1} x_{2}\langle 1 i k l+1\rangle+w_{2} x_{1}\langle 1 i k+1 l+1\rangle+w_{1} x_{2}\langle 1 i+1 k l+1\rangle+w_{1} w_{2}\langle 1 i+1 k+1 l+1\rangle \\
& +x_{2} y_{1}\langle 1 k l+1 j\rangle+x_{2} z_{1}\langle 1 k l+1 j+1\rangle+w_{2} y_{1}\langle 1 k+1 l+1 j\rangle+w_{2} z_{1}\langle 1 k+1 l+1 j+1\rangle \\
& +x_{1} x_{2} y_{1}\langle i k l+1 j\rangle+x_{1} x_{2} z_{1}\langle i k l+1 j+1\rangle+w_{2} x_{1} y_{1}\langle i k+1 l+1 j\rangle \\
& +w_{2} x_{1} z_{1}\langle i k+1 l+1 j+1\rangle+w_{1} x_{2} y_{1}\langle i+1 k l+1 j\rangle+w_{1} x_{2} z_{1}\langle i+1 k l+1 j+1\rangle \\
& +w_{1} w_{2} y_{1}\langle i+1 k+1 l+1 j\rangle+w_{1} w_{2} z_{1}\langle i+1 k+1 l+1 j+1\rangle \\
b= & x_{2} y_{1}\langle 1 i+1 k j\rangle+x_{2} z_{1}\langle 1 i+1 k j+1\rangle+y_{1} y_{2}\langle 1 i+1 l j\rangle+y_{2} z_{1}\langle 1 i+1 l j+1\rangle \\
& +y_{1} z_{2}\langle 1 i+1 l+1 j\rangle+z_{1} z_{2}\langle 1 i+1 l+1 j+1\rangle \\
c= & x_{2} y_{1}\langle 1 i k j\rangle+x_{2} z_{1}\langle 1 i k j+1\rangle+w_{2} y_{1}\langle 1 i k+1 j\rangle+w_{2} z_{1}\langle 1 i k+1 j+1\rangle+y_{1} y_{2}\langle 1 i l j\rangle \\
& +y_{2} z_{1}\langle 1 i l+1 j\rangle+y_{1} z_{2}\langle 1 i l+1 j\rangle+z_{1} z_{2}\langle 1 i l+1 j+1\rangle \\
d= & w_{1} y_{1}\langle 1 i+1 k+1 j\rangle+w_{1} z_{1}\langle 1 i+1 k+1 j+1\rangle \\
e= & x_{1} x_{2}\langle 1 i k l\rangle+w_{2} x_{1}\langle 1 i k+1 l\rangle+w_{1} x_{2}\langle 1 i+1 k l\rangle+w_{1} w_{2}\langle 1 i+1 k+1 l\rangle+x_{2} y_{1}\langle 1 k l j\rangle \\
& +x_{2} z_{1}\langle 1 k l j+1\rangle+w_{2} y_{1}\langle 1 k+1 l j\rangle+w_{2} z_{1}\langle 1 k+1 l j+1\rangle+x_{1} x_{2} y_{1}\langle i k l j\rangle \\
& +x_{1} x_{2} z_{1}\langle i k l j+1\rangle+w_{2} x_{1} y_{1}\langle i k+1 l j\rangle+w_{2} x_{1} z_{1}\langle i k+1 l j+1\rangle+w_{1} x_{2} y_{1}\langle i+1 k l j\rangle \\
& +w_{1} x_{2} z_{1}\langle i+1 k l j+1\rangle+w_{1} w_{2} y_{1}\langle i+1 k+1 l j\rangle+w_{1} w_{2} z_{1}\langle i+1 k+1 l j+1\rangle .
\end{aligned}
$$

We can calculate all forms for each pattern and the explicit form is written in the appendix. Then the full form of the 2-loop n-pt MHV log amplituhedron is

$\Omega\left[\log \left[\mathcal{A}_{\mathrm{MHV}}^{n \text {-pt 2-loop }}\right]\right]=\sum_{\substack{i, j, k, l=2,3, \cdots, n-1 \\ i<k<l<j}} \Omega_{i j k l}^{1}[\log ]+\sum_{i<k<j<l} \Omega_{i j k l}^{2}[\log ]+\cdots+\sum_{k<l<i<j} \Omega_{i j k l}^{13}[\log ]$. 
Then we can compare with this result and the non-planar sum of the double pentagon diagrams (4.8) and we checked that these results are corresponding up to at least 22-pt.

In the case of the 2-loop MHV amplitude, we can obtain the log of the amplitude from the canonical form on the well-defined space as (4.13). However, the important point is that in the case of the 3-loop or higher loop, we can not define the log of the amplitude as a canonical form on the well-defined space. This means that the 2-loop MHV case is a special that we can define the log of the amplitude geometrically.

\subsection{Square of the amplituhedron and positivity}

Next we consider the decomposition of the square of the 1-loop MHV amplituhedron. From (4.12), the square of the 1-loop MHV amplituhedron is decomposed into the amplituhedron and the log amplituhedron

$$
\begin{aligned}
\left(\mathcal{L}_{i}=D_{i} \cdot Z\right)= & \left(\mathcal{L}_{i}=D_{i} \cdot Z, \quad\left\langle D_{(1)} D_{(2)}\right\rangle>0\right) \\
& +\left(\mathcal{L}_{i}=D_{i} \cdot Z, \quad\left\langle D_{(1)} D_{(2)}\right\rangle<0\right)
\end{aligned}
$$

for $i=1,2$. We can see this decomposition directly from the canonical form. For example, the 4-point case, the canonical form of the amplitude and log of the amplitude is

$$
\begin{aligned}
\Omega[\mathcal{A}] & =\frac{d x_{1} d x_{2} d w_{1} d w_{2} d y_{1} d y_{2} d z_{1} d z_{2}}{x_{1} x_{2} w_{1} w_{2} y_{1} y_{2} z_{1} z_{2}} \frac{x_{1} z_{2}+x_{2} z_{1}+w_{1} y_{2}+w_{2} y_{1}}{\left\{\left(x_{1}-x_{2}\right)\left(z_{2}-z_{1}\right)+\left(w_{1}-w_{2}\right)\left(y_{2}-y_{1}\right)\right\}} \\
\Omega[\log \mathcal{A}] & =\frac{d x_{1} d x_{2} d w_{1} d w_{2} d y_{1} d y_{2} d z_{1} d z_{2}}{x_{1} x_{2} w_{1} w_{2} y_{1} y_{2} z_{1} z_{2}} \frac{-\left(x_{1} z_{1}+x_{2} z_{2}+w_{1} y_{1}+w_{2} y_{2}\right)}{\left\{\left(x_{1}-x_{2}\right)\left(z_{2}-z_{1}\right)+\left(w_{1}-w_{2}\right)\left(y_{2}-y_{1}\right)\right\}} .
\end{aligned}
$$

Then

$$
\Omega[\mathcal{A}]+\Omega[\log \mathcal{A}]=\frac{d x_{1} d x_{2} d w_{1} d w_{2} d y_{1} d y_{2} d z_{1} d z_{2}}{x_{1} x_{2} w_{1} w_{2} y_{1} y_{2} z_{1} z_{2}} .
$$

This is just the canonical form of the square of the 1-loop MHV amplituhedron (4.12). We can be confirmed that it holds for general $n$-point case from the explicit representation of the canonical form.

The interesting feature is that the numerator of the canonical form of the 2-loop MHV amplituhedron is the positive part of $\langle A B C D\rangle$ and the numerator of the log amplitude is the negative part. For example, the 4-pt case,

$$
\langle A B C D\rangle=\langle 1234\rangle\left\{x_{1} z_{2}+x_{2} z_{1}+w_{1} y_{2}+w_{2} y_{1}-\left(x_{1} z_{1}+x_{2} z_{2}+w_{1} y_{1}+w_{2} y_{2}\right)\right\} .
$$

From the condition that $(A, B)$ and $(C, D)$ are the 1-loop MHV amplituhedron, we can see that

$$
\langle 1234\rangle, x_{1}, x_{2}, w_{1}, w_{2}, \cdots, z_{2}>0 .
$$

Then $\langle A B C D\rangle$ is decomposed to

$$
\langle A B C D\rangle=A^{+}+A^{-}
$$

where

$$
A^{+}=\langle 1234\rangle\left(x_{1} z_{2}+x_{2} z_{1}+w_{1} y_{2}+w_{2} y_{1}\right), \quad A^{-}=-\langle 1234\rangle\left(x_{1} z_{1}+x_{2} z_{2}+w_{1} y_{1}+w_{2} y_{2}\right)
$$


and $A^{+}$is positive, $A^{-}$is negative. From (4.21),

$$
\begin{aligned}
\Omega[\mathcal{A}] & =\frac{d x_{1} d x_{2} d w_{1} d w_{2} d y_{1} d y_{2} d z_{1} d z_{2}}{x_{1} x_{2} w_{1} w_{2} y_{1} y_{2} z_{1} z_{2}} \frac{A^{+}}{\langle A B C D\rangle} \\
\Omega[\log \mathcal{A}] & =\frac{d x_{1} d x_{2} d w_{1} d w_{2} d y_{1} d y_{2} d z_{1} d z_{2}}{x_{1} x_{2} w_{1} w_{2} y_{1} y_{2} z_{1} z_{2}} \frac{A^{-}}{\langle A B C D\rangle},
\end{aligned}
$$

we use the relation

$$
\langle 1234\rangle\left\{\left(x_{1}-x_{2}\right)\left(z_{2}-z_{1}\right)+\left(w_{1}-w_{2}\right)\left(y_{2}-y_{1}\right)\right\}=\langle A B C D\rangle .
$$

From the $n$-point forms of the amplitude and the log amplitude, we can see that this holds for general $n$-point case. $\langle A B C D\rangle$ is decomposed into the positive and negative parts even for the $n$-pt case. For example, the pattern (1) for (3.41),

$$
\langle A B C D\rangle=a z_{2}-b w_{1}-c x_{1}-d w_{2}+e y_{2}
$$

where $a, b, c, d, e$ are defined as (4.18) and these are positive. Then the positive and negative parts is

$$
A^{+}=a z_{2}+e y_{2}, \quad A^{-}=-\left(b w_{1}+c x_{1}+d w_{2}\right) .
$$

The canonical form of the 2-loop amplitude and the log of the amplitude for this pattern (1) is

$$
\begin{aligned}
\Omega_{i j k l}^{1}[\mathcal{A}] & =\frac{d x_{1} d x_{2} d w_{1} d w_{2} d y_{1} d y_{2} d z_{1} d z_{2}}{x_{1} x_{2} w_{1} w_{2} y_{1} y_{2} z_{1} z_{2}} \frac{A^{+}}{\langle A B C D\rangle} \\
\Omega_{i j k l}^{1}[\log \mathcal{A}] & =\frac{d x_{1} d x_{2} d w_{1} d w_{2} d y_{1} d y_{2} d z_{1} d z_{2}}{x_{1} x_{2} w_{1} w_{2} y_{1} y_{2} z_{1} z_{2}} \frac{A^{-}}{\langle A B C D\rangle}
\end{aligned}
$$

and we can see that this holds for all another patterns of (3.41). From this result and $A^{+}>0$, the form of the $n$-pt 2-loop MHV amplituhedron is positive. Addition to this, in the form of the $\log$ amplitude, $A^{-}<0$ and $\langle A B C D\rangle<0$. Then the log of the amplitude is also positive. The positivity of the canonical form is related to the existence of a "dual amplituhedron" [8]. Then this is the another prove of the positivity of the canonical form directly.

\section{Conclusion}

In this work, we found the direct triangulation of the 2-loop MHV amplituhedron from the sign flip definition. These cells are non-redundant cells and live directly in $(A B),(C D)$ space. Unlike the BCFW case, there is no reference to any tree amplitudes. We have seen that the $n$-point 2-loop MHV amplituhedron is triangulated into the $\frac{1}{4}(n-3)^{2}(n-2)^{2}$ cells and these are divided into 13 groups. These have spurious poles but in the sum of these cells, the spurious poles are canceled and only remain the physical poles. We checked these results are consistent with the local representation and the BCFW. 
We obtained the $n$-point 2-loop MHV log amplitude from the geometry that constructed from the two 1-loop MHV amplituhedron and a negative constraint. However, this 2-loop MHV case is a special that we can define the log of the amplitude geometrically. The 3-loop or higher loop case, we can not define the log of the amplitude as a canonical form on the well-defined space.

In the case of the 1-loop MHV amplituhedron, the triangulation from the sign flips corresponds to the BCFW triangulation. However, in the 2-loop MHV case, this triangulation doesn't correspond to the BCFW. Then the question is how to interpret this triangulation as the recursion relation.

The natural generalization is to go to the higher loop MHV amplituhedron. For example, the 3-loop MHV case, we have three 1-loop amplituhedron $(A B),(C D),(E F)$ and three constraints $\langle A B C D\rangle>0,\langle A B E F\rangle>0,\langle C D E F\rangle>0$. In general $L$-loop MHV amplituhedron, there are $L$ 1-loop MHV amplituhedron and $\frac{1}{2} L(L-1)$ constraints. Is it possible to obtain the general canonical form of the 3 or general $L$-loop MHV amplituhedron from the sign flip triangulation?

Another generalization is to go to a higher $k$ amplituhedron. However, it is difficult to generalize this triangulation to the general $\mathrm{N}^{k} \mathrm{MHV}$ amplituhedron. Because in the MHV case, we can use the isomorphism between the $k=2, m=2$ tree amplituhedron and the 1loop MHV amplituhedron to obtain the direct triangulation from sign flips. However, there is no isomorphism between the $m=2$ amplituhedron and the $\mathrm{N}^{k} \mathrm{MHV}$ loop amplituhedron. Then the future work is that to avoid using the isomorphism, we need to consider the direct relation between the triangulation of the $m=4$ amplituhedron and the sign flip patterns.

\section{Acknowledgments}

We thank Jaroslav Trnka for first suggesting the problem, for numerous stimulating discussions and comments on the draft. This work was supported through the hospitality of the Center for Quantum Mathematics and Physics (QMAP), Department of Physics, University of California, Davis. This work is supported by the FY2017 Course-by-Course Education Program to Cultivate Researchers in Physical Sciences with Broad Perspectives, SOKENDAI.

\section{A Explicit calculation of the 2-loop 5-point MHV amplitude}

The case of $(2,3) \times(2,3)$,

$$
\left\{\begin{array} { l } 
{ Z _ { A } = Z _ { 1 } + x _ { 1 } Z _ { 2 } + w _ { 1 } Z _ { 3 } } \\
{ Z _ { B } = - Z _ { 1 } + y _ { 1 } Z _ { 3 } + z _ { 1 } Z _ { 4 } }
\end{array} \quad \left\{\begin{array}{l}
Z_{C}=Z_{1}+x_{2} Z_{2}+w_{2} Z_{3} \\
Z_{D}=-Z_{1}+y_{2} Z_{3}+z_{2} Z_{4}
\end{array}\right.\right.
$$

Therefore it is same as 4-pt case. $C$-matrix is (3.17) and the form is (3.27).

Next the case of $(3,4) \times(3,4)$,

$$
\left\{\begin{array} { l } 
{ Z _ { A } = Z _ { 1 } + x _ { 1 } Z _ { 3 } + w _ { 1 } Z _ { 4 } } \\
{ Z _ { B } = - Z _ { 1 } + y _ { 1 } Z _ { 4 } + z _ { 1 } Z _ { 5 } }
\end{array} \quad \left\{\begin{array}{l}
Z_{C}=Z_{1}+x_{2} Z_{3}+w_{2} Z_{4} \\
Z_{D}=-Z_{1}+y_{2} Z_{4}+z_{2} Z_{5}
\end{array}\right.\right.
$$


Then

$$
\langle A B C D\rangle=\langle 1345\rangle\left\{\left(x_{1}-x_{2}\right)\left(y_{1} z_{2}-y_{2} z_{1}\right)+\left(z_{1}-z_{2}\right)\left(w_{1} x_{2}-w_{2} x_{1}\right)\right\}
$$

It is almost same as the case of $(2,3) \times(2,3)$. The only difference is $\langle 1345\rangle$, thus there are 8 forms and the sum of these forms is

$$
\begin{aligned}
\Omega_{3434}= & \frac{d x_{1} d x_{2} d w_{1} d w_{2} d y_{1} d y_{2} d z_{1} d z_{2}}{x_{1} x_{2} w_{1} w_{2} y_{1} y_{2} z_{1} z_{2}} \frac{\langle 1345\rangle}{\langle A B C D\rangle}\left(x_{1} y_{1} z_{2}+x_{2} y_{2} z_{1}+x_{2} w_{1} z_{1}+x_{1} w_{2} z_{2}\right) \\
= & \frac{\langle 1345\rangle^{3}\left\langle A B d^{2} A\right\rangle\left\langle A B d^{2} B\right\rangle\left\langle C D d^{2} C\right\rangle\left\langle C D d^{2} D\right\rangle}{\langle A B 13\rangle\langle A B 15\rangle\langle A B 34\rangle\langle A B 45\rangle\langle A B C D\rangle\langle C D 13\rangle\langle C D 15\rangle\langle C D 34\rangle\langle C D 45\rangle} \\
& \times\{\langle A B 45\rangle\langle C D 13\rangle+\langle A B 34\rangle\langle C D 15\rangle+\langle A B 15\rangle\langle C D 34\rangle+\langle A B 13\rangle\langle C D 45\rangle\}
\end{aligned}
$$

The case of $(2,3) \times(2,4)$, two 1-loop amplituhedron are parametrized as

$$
\left\{\begin{array} { l } 
{ Z _ { A } = Z _ { 1 } + x _ { 1 } Z _ { 2 } + w _ { 1 } Z _ { 3 } } \\
{ Z _ { B } = - Z _ { 1 } + y _ { 1 } Z _ { 3 } + z _ { 1 } Z _ { 4 } }
\end{array} \quad \left\{\begin{array}{l}
Z_{C}=Z_{1}+x_{2} Z_{2}+w_{2} Z_{3} \\
Z_{D}=-Z_{1}+y_{2} Z_{4}+z_{2} Z_{5}
\end{array}\right.\right.
$$

In view of the $Y=C \cdot Z$ description, the $C$-matrix is

$$
C=\left(\begin{array}{ccccc}
1 & x_{1} & w_{1} & 0 & 0 \\
-1 & 0 & y_{1} & z_{1} & 0 \\
1 & x_{2} & w_{2} & 0 & 0 \\
-1 & 0 & 0 & y_{2} & z_{2}
\end{array}\right)
$$

The constraint is

$$
\begin{aligned}
\langle A B C D\rangle= & \left(x_{1}-x_{2}\right)\left\{y_{1} y_{2}\langle 1234\rangle+y_{1} z_{2}\langle 1235\rangle+z_{1} z_{2}\langle 1245\rangle\right\} \\
& +\left(x_{1} w_{2}-x_{2} w_{1}\right)\left\{\left(y_{2}-z_{1}\right)\langle 1234\rangle+z_{2}\langle 1235\rangle-z_{1} z_{2}\langle 2345\rangle\right\} \\
& +\left(w_{1}-w_{2}\right) z_{1} z_{2}\langle 1345\rangle
\end{aligned}
$$

From $\langle A B C D\rangle>0$,

$$
\begin{aligned}
x_{1} & >x_{2}-\frac{\left(x_{1} w_{2}-x_{2} w_{1}\right)\left\{\left(y_{2}-z_{1}\right)\langle 1234\rangle+z_{2}\langle 1235\rangle-z_{1} z_{2}\langle 2345\rangle\right\}+\left(w_{1}-w_{2}\right) z_{1} z_{2}\langle 1345\rangle}{y_{1} y_{2}\langle 1234\rangle+y_{1} z_{2}\langle 1235\rangle+z_{1} z_{2}\langle 1245\rangle} \\
& =x_{2}-a
\end{aligned}
$$

The region of $x_{1}$ is depends on the sign of $a$. When $a<0$,

$$
x_{1}>x_{2}-a, w_{2}<w_{1}-\frac{\left(x_{1} w_{2}-x_{2} w_{1}\right)\left\{\left(y_{2}-z_{1}\right)\langle 1234\rangle+z_{2}\langle 1235\rangle-z_{1} z_{2}\langle 2345\rangle\right\}}{z_{1} z_{2}\langle 1345\rangle}=w_{1}-b
$$


Similarly, the region of $w_{1}$ is depends on the sign of $b$. When $b<0$, there are 2 cases that

$$
\left\{\begin{array}{c}
w_{1}<w_{2}-b, \text { and } x_{2}<\frac{w_{2}}{w_{1}} x_{1}, \quad z_{1}>\frac{y_{2}\langle 1234\rangle+z_{2}\langle 1235\rangle}{\langle 1234\rangle+z_{2}\langle 2345\rangle}=c \\
\text { or } \\
w_{1}<w_{2}-b, \text { and } x_{2}>\frac{w_{2}}{w_{1}} x_{1}, \quad z_{1}<c
\end{array}\right.
$$

There are 8 cases depending on the signs of a,b. The forms for these cases can be obtained similarly as 4-point case,

$$
\begin{aligned}
\Omega_{1} & =\frac{1}{x_{1}-x_{2}+a}\left(\frac{1}{x_{2}}-\frac{1}{x_{2}-\frac{w_{2}}{w_{1}} x_{1}}\right)\left(\frac{1}{w_{1}}-\frac{1}{w_{1}-w_{2}+b}\right) \frac{1}{w_{2}} \frac{1}{y_{1}} \frac{1}{y_{2}} \frac{1}{z_{1}-c} \frac{1}{z_{2}} \\
\Omega_{2} & =\frac{1}{x_{1}-x_{2}+a} \frac{1}{x_{2}-\frac{w_{2}}{w_{1}} x_{1}}\left(\frac{1}{w_{1}}-\frac{1}{w_{1}-w_{2}+b}\right) \frac{1}{w_{2}} \frac{1}{y_{1}} \frac{1}{y_{2}}\left(\frac{1}{z_{1}}-\frac{1}{z_{1}-c}\right) \frac{1}{z_{2}} \\
\Omega_{3} & =\frac{1}{x_{1}-x_{2}+a}\left(\frac{1}{x_{2}}-\frac{1}{x_{2}-\frac{w_{2}}{w_{1}} x_{1}}\right) \frac{1}{w_{1}} \frac{1}{w_{2}-w_{1}-b} \frac{1}{y_{1}} \frac{1}{y_{2}}\left(\frac{1}{z_{1}}-\frac{1}{z_{1}-c}\right) \frac{1}{z_{2}} \\
\Omega_{4} & =\frac{1}{x_{1}-x_{2}+a} \frac{1}{x_{2}-\frac{w_{2}}{w_{1}} x_{1}} \frac{1}{w_{1}} \frac{1}{w_{2}-w_{1}-b} \frac{1}{y_{1}} \frac{1}{y_{2}} \frac{1}{z_{1}-c} \frac{1}{z_{2}} \\
\Omega_{5} & =\left(\frac{1}{x_{2}}-\frac{1}{x_{2}-x_{1}-a}\right) \frac{1}{x_{1}-\frac{w_{1}}{w_{2}} x_{2}} \frac{1}{w_{1}-w_{2}+b} \frac{1}{w_{2}} \frac{1}{y_{1}} \frac{1}{y_{2}} \frac{1}{z_{1}-c} \frac{1}{z_{2}} \\
\Omega_{6} & =\left(\frac{1}{x_{2}}-\frac{1}{x_{2}-x_{1}-a}\right)\left(\frac{1}{x_{1}}-\frac{1}{x_{1}-\frac{w_{1}}{w_{2}} x_{2}}\right) \frac{1}{w_{1}-w_{2}+b} \frac{1}{w_{2}} \frac{1}{y_{1}} \frac{1}{y_{2}}\left(\frac{1}{z_{1}}-\frac{1}{z_{1}-c}\right) \frac{1}{z_{2}} \\
\Omega_{7} & =\left(\frac{1}{x_{2}}-\frac{1}{x_{2}-x_{1}-a}\right) \frac{1}{x_{1}-\frac{w_{1}}{w_{2}} x_{2}} \frac{1}{w_{1}}\left(\frac{1}{w_{2}}-\frac{1}{w_{2}-w_{1}-b}\right) \frac{1}{y_{1}} \frac{1}{y_{2}}\left(\frac{1}{z_{1}}-\frac{1}{z_{1}-c}\right) \frac{1}{z_{2}} \\
\Omega_{8} & =\left(\frac{1}{x_{2}}-\frac{1}{x_{2}-x_{1}-a}\right)\left(\frac{1}{x_{1}}-\frac{1}{x_{1}-\frac{w_{1}}{w_{2}} x_{2}}\right) \frac{1}{w_{1}}\left(\frac{1}{w_{2}}-\frac{1}{w_{2}-w_{1}-b}\right) \frac{1}{y_{1}} \frac{1}{y_{2}} \frac{1}{z_{1}-c} \frac{1}{z_{2}}
\end{aligned}
$$

For

$$
\begin{aligned}
a & =\frac{\left(x_{1} w_{2}-x_{2} w_{1}\right)\left\{\left(y_{2}-z_{1}\right)\langle 1234\rangle+z_{2}\langle 1235\rangle-z_{1} z_{2}\langle 2345\rangle\right\}+\left(w_{1}-w_{2}\right) z_{1} z_{2}\langle 1345\rangle}{y_{1} y_{2}\langle 1234\rangle+y_{1} z_{2}\langle 1235\rangle+z_{1} z_{2}\langle 1245\rangle} \\
b & =\frac{\left(x_{1} w_{2}-x_{2} w_{1}\right)\left\{\left(y_{2}-z_{1}\right)\langle 1234\rangle+z_{2}\langle 1235\rangle-z_{1} z_{2}\langle 2345\rangle\right\}}{z_{1} z_{2}\langle 1345\rangle} \\
c & =\frac{y_{2}\langle 1234\rangle+z_{2}\langle 1235\rangle}{\langle 1234\rangle+z_{2}\langle 2345\rangle}
\end{aligned}
$$

Then sum of these 8 forms is

$$
\begin{aligned}
\Omega_{2324}= & \frac{d x_{1} d x_{2} d w_{1} d w_{2} d y_{1} d y_{2} d z_{1} d z_{2}}{x_{1} x_{2} w_{1} w_{2} y_{1} y_{2} z_{1} z_{2}} \frac{1}{\langle A B C D\rangle}\left\{\langle 1234\rangle\left(x_{1} w_{2} y_{2}+x_{1} y_{1} y_{2}+x_{2} w_{1} z_{1}\right)\right. \\
& \left.+\langle 1235\rangle x_{1} z_{2}\left(w_{2}+y_{1}\right)+z_{1} z_{2}\left(\langle 1345\rangle w_{1}+\langle 1245\rangle x_{1}+\langle 2345\rangle x_{2} w_{1}\right)\right\}
\end{aligned}
$$


Rewrite it into the momentum twistor,

$$
\begin{aligned}
& \Omega_{2324}=\frac{\left\langle 123 A_{3}\right\rangle\left\langle 123 C_{4}\right\rangle\left\langle A B d^{2} A\right\rangle\left\langle A B d^{2} B\right\rangle\left\langle C D d^{2} C\right\rangle\left\langle C D d^{2} D\right\rangle}{\left\{\begin{array}{c}
\langle A B 12\rangle\langle A B 13\rangle^{2}\langle A B 14\rangle\langle A B 23\rangle\langle A B 34\rangle \\
\times\langle A B C D\rangle\langle C D 12\rangle\langle C D 13\rangle\langle C D 14\rangle\langle C D 15\rangle\langle C D 23\rangle\langle C D 45\rangle
\end{array}\right\}} \\
& \times\left\{\left\langle 123 A_{3}\right\rangle(\langle A B 12\rangle\langle C D 13\rangle\langle C D 45\rangle+\langle A B 15\rangle\langle C D 14\rangle\langle C D 23\rangle)\right. \\
& \left\langle 123 C_{4}\right\rangle(\langle A B 13\rangle\langle A B 34\rangle\langle C D 12\rangle+\langle A B 13\rangle\langle A B 14\rangle\langle C D 23\rangle) \\
& -\langle 1235\rangle\langle A B 13\rangle\langle A B 14\rangle\langle C D 14\rangle\langle C D 23\rangle-\langle 2345\rangle\langle A B 12\rangle\langle A B 13\rangle\langle C D 12\rangle\langle C D 14\rangle\} \text {. }
\end{aligned}
$$

We use these symbols

$$
\begin{aligned}
(A B) \cap(1 i i+1)=-Z_{1}\langle i i+1 A B\rangle-Z_{i}\langle i+1 A B 1\rangle-Z_{i+1}\langle A B 1 i\rangle & \equiv A_{i} \\
(C D) \cap(1 i i+1)=-Z_{1}\langle i i+1 C D\rangle-Z_{i}\langle i+1 C D 1\rangle-Z_{i+1}\langle C D 1 i\rangle & \equiv C_{i}
\end{aligned}
$$

The case of $(2,4) \times(3,4)$,

$$
\left\{\begin{array} { l } 
{ Z _ { A } = Z _ { 1 } + x _ { 1 } Z _ { 2 } + w _ { 1 } Z _ { 3 } } \\
{ Z _ { B } = - Z _ { 1 } + y _ { 1 } Z _ { 4 } + z _ { 1 } Z _ { 5 } }
\end{array} \quad \left\{\begin{array}{l}
Z_{C}=Z_{1}+x_{2} Z_{3}+w_{2} Z_{4} \\
Z_{D}=-Z_{1}+y_{2} Z_{4}+z_{2} Z_{5}
\end{array}\right.\right.
$$

$C$-matrix is

$$
\begin{aligned}
C= & \left(\begin{array}{ccccc}
1 & x_{1} & w_{1} & 0 & 0 \\
-1 & 0 & 0 & y_{1} & z_{1} \\
1 & 0 & x_{2} & w_{2} & 0 \\
-1 & 0 & 0 & y_{2} & z_{2}
\end{array}\right) \\
\langle A B C D\rangle= & \left(z_{2}-z_{1}\right)\left(\langle 1345\rangle w_{1} w_{2}+\langle 1235\rangle x_{1} x_{2}+\langle 1245\rangle x_{1} w_{2}\right) \\
& +\left(z_{1} y_{2}-z_{2} y_{1}\right)\left\{\langle 1345\rangle\left(x_{2}-w_{1}\right)-\langle 1245\rangle x_{1}+\langle 2345\rangle x_{1} x_{2}\right\} \\
& +\left(y_{2}-y_{1}\right)\langle 1234\rangle x_{1} x_{2}
\end{aligned}
$$

From $\langle A B C D\rangle>0$,

$$
\begin{aligned}
z_{2} & >z_{1}-\frac{\left(z_{1} y_{2}-z_{2} y_{1}\right)\left\{\langle 1345\rangle\left(x_{2}-w_{1}\right)-\langle 1245\rangle x_{1}+\langle 2345\rangle x_{1} x_{2}\right\}+\left(y_{2}-y_{1}\right)\langle 1234\rangle x_{1} x_{2}}{x_{1} x_{2}\langle 1234\rangle} \\
& =z_{1}-a
\end{aligned}
$$

The region of $z_{2}$ is depends on the sign of $a$. When $a<0$,

$$
z_{2}>z_{1}-a, \text { and } y_{2}<y_{1}-\frac{\left(z_{1} y_{2}-z_{2} y_{1}\right)\left\{\langle 1345\rangle\left(x_{2}-w_{1}\right)-\langle 1245\rangle x_{1}+\langle 2345\rangle x_{1} x_{2}\right\}}{x_{1} x_{2}\langle 1234\rangle}=y_{1}-b
$$

Similarly, the region of $y_{2}$ is depends on the sign of $b$. When $b<0$, there are 2 cases that

$$
y_{2}<y_{1}-b, \text { and }\left\{\begin{array}{cc}
z_{1}>\frac{y_{1}}{y_{2}} z_{2}, & x_{2}<\frac{w_{1}\langle 1345\rangle+x_{1}\langle 1245\rangle}{x_{1}\langle 2345\rangle+\langle 1345\rangle}=c \\
\text { or } & \\
z_{1}<\frac{y_{1}}{y_{2}} z_{2}, & x_{2}>c
\end{array}\right.
$$


There are 8 cases depending on the signs of a,b. Then the forms for these cases are

$$
\begin{aligned}
& \Omega_{1}=\frac{1}{x_{1}}\left(\frac{1}{x_{2}}-\frac{1}{x_{2}-c}\right) \frac{1}{w_{1}} \frac{1}{w_{2}} \frac{1}{y_{1}}\left(\frac{1}{y_{2}}-\frac{1}{y_{2}-y_{1}+b}\right) \frac{1}{z_{1}-\frac{y_{1} z_{2}}{y_{2}}} \frac{1}{z_{2}-z_{1}+a} \\
& \Omega_{2}=\frac{1}{x_{1}} \frac{1}{x_{2}-c} \frac{1}{w_{1}} \frac{1}{w_{2}} \frac{1}{y_{1}}\left(\frac{1}{y_{2}}-\frac{1}{y_{2}-y_{1}+b}\right)\left(\frac{1}{z_{1}}-\frac{1}{\left.z_{1}-\frac{y_{1} z_{2}}{y_{2}}\right)} \frac{1}{z_{2}-z_{1}+a}\right. \\
& \Omega_{3}=\frac{1}{x_{1}} \frac{1}{x_{2}-c} \frac{1}{w_{1}} \frac{1}{w_{2}} \frac{1}{y_{1}-y_{2}-b} \frac{1}{y_{2}} \frac{1}{z_{1}-\frac{y_{1} z_{2}}{y_{2}} \frac{1}{z_{2}-z_{1}+a}} \\
& \Omega_{4}=\frac{1}{x_{1}}\left(\frac{1}{x_{2}}-\frac{1}{x_{2}-c}\right) \frac{1}{w_{1}} \frac{1}{w_{2}} \frac{1}{y_{1}} \frac{1}{y_{2}-y_{1}+b}\left(\frac{1}{z_{1}}-\frac{1}{z_{1}-\frac{y_{1}}{y_{2}} z_{2}}\right) \frac{1}{z_{2}-z_{1}+a} \\
& \Omega_{5}=\frac{1}{x_{1}}\left(\frac{1}{x_{2}}-\frac{1}{x_{2}-c}\right) \frac{1}{w_{1}} \frac{1}{w_{2}} \frac{1}{y_{1}} \frac{1}{y_{2}-y_{1}+b}\left(\frac{1}{z_{1}}-\frac{1}{z_{1}-\frac{y_{1}}{y_{2}} z_{2}}\right)\left(\frac{1}{z_{2}}-\frac{1}{z_{2}-z_{1}+a}\right) \\
& \Omega_{6}=\frac{1}{x_{1}} \frac{1}{x_{2}-c} \frac{1}{w_{1}} \frac{1}{w_{2}} \frac{1}{y_{1}} \frac{1}{y_{2}-y_{1}+b}\left(\frac{1}{z_{1}}-\frac{1}{z_{1}-\frac{y_{1}}{y_{2}} z_{2}}\right) \frac{1}{z_{2}-z_{1}+a} \\
& \Omega_{7}=\frac{1}{x_{1}} \frac{1}{x_{2}-c} \frac{1}{w_{1}} \frac{1}{w_{2}}\left(\frac{1}{y_{1}}-\frac{1}{y_{1}-y_{2}-b}\right) \frac{1}{y_{2}}\left(\frac{1}{z_{1}}-\frac{1}{z_{1}-\frac{y_{1}}{y_{2}} z_{2}}\right)\left(\frac{1}{z_{2}}-\frac{1}{z_{2}-z_{1}+a}\right) \\
& \Omega_{8}=\frac{1}{x_{1}}\left(\frac{1}{x_{2}}-\frac{1}{x_{2}-c}\right) \frac{1}{w_{1}} \frac{1}{w_{2}}\left(\frac{1}{y_{1}}-\frac{1}{y_{1}-y_{2}-b}\right) \frac{1}{y_{2}}\left(\frac{1}{z_{1}}-\frac{1}{z_{1}-\frac{y_{1}}{y_{2}}}\right) \frac{1}{z_{2}-z_{1}+a}
\end{aligned}
$$

For

$$
\begin{aligned}
a & =\frac{\left(z_{1} y_{2}-z_{2} y_{1}\right)\left\{\langle 1345\rangle\left(x_{2}-w_{1}\right)-\langle 1245\rangle x_{1}+\langle 2345\rangle x_{1} x_{2}\right\}+\left(y_{2}-y_{1}\right)\langle 1234\rangle x_{1} x_{2}}{x_{1} x_{2}\langle 1234\rangle} \\
b & =\frac{\left(z_{1} y_{2}-z_{2} y_{1}\right)\left\{\langle 1345\rangle\left(x_{2}-w_{1}\right)-\langle 1245\rangle x_{1}+\langle 2345\rangle x_{1} x_{2}\right\}}{x_{1} x_{2}\langle 1234\rangle} \\
c & =\frac{w_{1}\langle 1345\rangle+x_{1}\langle 1245\rangle}{x_{1}\langle 2345\rangle+\langle 1345\rangle}
\end{aligned}
$$

Then sum of these 8 forms is

$$
\begin{aligned}
\Omega_{2434}= & \frac{d x_{1} d x_{2} d w_{1} d w_{2} d y_{1} d y_{2} d z_{1} d z_{2}}{x_{1} x_{2} w_{1} w_{2} y_{1} y_{2} z_{1} z_{2}} \frac{1}{\langle A B C D\rangle}\left\{\langle 1345\rangle\left(w_{1} w_{2} z_{2}+w_{1} y_{1} z_{2}+x_{2} y_{2} z_{1}\right)\right. \\
& \left.+\langle 1235\rangle x_{1} x_{2} z_{2}+\langle 1245\rangle\left(x_{1} w_{2} z_{2}+x_{1} y_{1} z_{2}\right)+\langle 1234\rangle x_{1} x_{2} y_{2}+\langle 2345\rangle x_{1} x_{2} y_{2} z_{1}\right\}
\end{aligned}
$$

In the momentum twistor,

$$
\begin{aligned}
\Omega_{2434}= & \frac{\left\langle 123 A_{4}\right\rangle\left\langle 134 C_{4}\right\rangle\left\langle A B d^{2} A\right\rangle\left\langle A B d^{2} B\right\rangle\left\langle C D d^{2} C\right\rangle\left\langle C D d^{2} D\right\rangle}{\left\{\begin{array}{c}
\langle A B 12\rangle\langle A B 13\rangle\langle A B 14\rangle\langle A B 15\rangle\langle A B 23\rangle\langle A B 45\rangle \\
\times\langle A B C D\rangle\langle C D 13\rangle\langle C D 14\rangle^{2}\langle C D 15\rangle\langle C D 34\rangle\langle C D 45\rangle
\end{array}\right\}} \\
& \times\left\{\begin{array}{c}
\left\langle 123 A_{4}\right\rangle(\langle A B 45\rangle\langle C D 13\rangle\langle C D 14\rangle+\langle A B 15\rangle\langle C D 34\rangle\langle C D 14\rangle) \\
\end{array}\right. \\
& \left.-\left\langle 345 A_{2}\right\rangle\langle A B 14\rangle\langle C D 14\rangle\langle C D 15\rangle+\left\langle 123 C_{4}\right\rangle\langle C D 14\rangle\langle A B 45\rangle\langle A B 13\rangle\right\}
\end{aligned}
$$


The case of $(2,3) \times(3,4)$,

$$
\left\{\begin{array} { l } 
{ Z _ { A } = Z _ { 1 } + x _ { 1 } Z _ { 2 } + w _ { 1 } Z _ { 3 } } \\
{ Z _ { B } = - Z _ { 1 } + y _ { 1 } Z _ { 3 } + z _ { 1 } Z _ { 4 } }
\end{array} \quad \left\{\begin{array}{l}
Z_{C}=Z_{1}+x_{2} Z_{3}+w_{2} Z_{4} \\
Z_{D}=-Z_{1}+y_{2} Z_{4}+z_{2} Z_{5}
\end{array}\right.\right.
$$

$C$-matrix is

$$
\begin{aligned}
C= & \left(\begin{array}{ccccc}
1 & x_{1} & w_{1} & 0 & 0 \\
-1 & 0 & y_{1} & z_{1} & 0 \\
1 & 0 & x_{2} & w_{2} & 0 \\
-1 & 0 & 0 & y_{2} & z_{2}
\end{array}\right) \\
\langle A B C D\rangle= & \left(y_{1} w_{2}-z_{1} x_{2}\right)\left(\langle 1345\rangle z_{2}+\langle 1234\rangle x_{1}+\langle 2345\rangle x_{1} z_{2}\right) \\
& +z_{2}\left(z_{1}+w_{2}\right)\left(\langle 1345\rangle w_{1}+\langle 1245\rangle x_{1}\right)+x_{1}\left(y_{1}+x_{2}\right)\left(\langle 1235\rangle z_{2}+\langle 1234\rangle y_{2}\right)
\end{aligned}
$$

In this case, from $\langle A B C D\rangle>0$,

$$
y_{1} w_{2}-z_{1} x_{2}>-\frac{z_{2}\left(z_{1}+w_{2}\right)\left(\langle 1345\rangle w_{1}+\langle 1245\rangle x_{1}\right)+x_{1}\left(y_{1}+x_{2}\right)\left(\langle 1235\rangle z_{2}+\langle 1234\rangle y_{2}\right)}{\langle 1345\rangle z_{2}+\langle 1234\rangle x_{1}+\langle 2345\rangle x_{1} z_{2}}=-a
$$

However, from $x_{1}, x_{2}, w_{1}, w_{2}, y_{1}, y_{2}, z_{1}, z_{2}>0, a>0$. Therefore

$$
z_{1}<\frac{w_{2} y_{1}}{x_{2}}+\frac{a}{x_{2}}
$$

Then the form is

$$
\begin{aligned}
\Omega_{2334}= & \frac{d x_{1} d x_{2} d w_{1} d w_{2} d y_{1} d y_{2} d z_{1} d z_{2}}{x_{1} x_{2} w_{1} w_{2} y_{1} y_{2} z_{2}}\left(\frac{1}{z_{1}}-\frac{1}{z_{1}-\frac{w_{2} y_{1}}{x_{2}}-\frac{a}{x_{2}}}\right) \\
= & \frac{d x_{1} d x_{2} d w_{1} d w_{2} d y_{1} d y_{2} d z_{1} d z_{2}}{x_{1} x_{2} w_{1} w_{2} y_{1} y_{2} z_{1} z_{2}} \frac{1}{\langle A B C D\rangle}\left\{w_{2} y_{1}\left(\langle 1345\rangle z_{2}+\langle 1234\rangle x_{1}+\langle 2345\rangle x_{1} z_{2}\right)\right. \\
& \left.+z_{2}\left(z_{1}+w_{2}\right)\left(\langle 1345\rangle w_{1}+\langle 1245\rangle x_{1}\right)+x_{1}\left(y_{1}+x_{2}\right)\left(\langle 1235\rangle z_{2}+\langle 1234\rangle y_{2}\right)\right\}
\end{aligned}
$$

We can write it in the momentum twistor space,

$$
\begin{aligned}
& \Omega_{2334}=\frac{\left\langle 123 A_{3}\right\rangle\left\langle 134 C_{4}\right\rangle\left\langle A B d^{2} A\right\rangle\left\langle A B d^{2} B\right\rangle\left\langle C D d^{2} C\right\rangle\left\langle C D d^{2} D\right\rangle}{\left\{\begin{array}{c}
\langle A B 12\rangle\langle A B 13\rangle^{2}\langle A B 14\rangle\langle A B 23\rangle\langle A B 34\rangle \\
\times\langle A B C D\rangle\langle C D 13\rangle\langle C D 14\rangle^{2}\langle C D 15\rangle\langle C D 34\rangle\langle C D 45\rangle
\end{array}\right\}} \\
& \times\left\{\langle A B 13\rangle\left\langle 123 C_{4}\right\rangle\left\langle C D 4 A_{3}\right\rangle-\langle A B 13\rangle\langle A B 14\rangle\langle C D 13\rangle\left\langle 234 C_{4}\right\rangle\right. \\
& \left.+\langle C D 14\rangle\left\langle 145 A_{2}\right\rangle\left\langle C D 3 A_{3}\right\rangle-\langle A B 14\rangle\langle A B 23\rangle\langle C D 13\rangle\langle C D 14\rangle\langle 1345\rangle\right\}
\end{aligned}
$$


The case of $(2,4) \times(2,4)$,

$$
\left\{\begin{array} { l } 
{ Z _ { A } = Z _ { 1 } + x _ { 1 } Z _ { 2 } + w _ { 1 } Z _ { 3 } } \\
{ Z _ { B } = - Z _ { 1 } + y _ { 1 } Z _ { 4 } + z _ { 1 } Z _ { 5 } }
\end{array} \quad \left\{\begin{array}{l}
Z_{C}=Z_{1}+x_{2} Z_{2}+w_{2} Z_{3} \\
Z_{D}=-Z_{1}+y_{2} Z_{4}+z_{2} Z_{5}
\end{array}\right.\right.
$$

$C$-matrix is

$$
\begin{aligned}
C= & \left(\begin{array}{ccccc}
1 & x_{1} & w_{1} & 0 & 0 \\
-1 & 0 & 0 & y_{1} & z_{1} \\
1 & x_{2} & w_{2} & 0 & 0 \\
-1 & 0 & 0 & y_{2} & z_{2}
\end{array}\right) \\
\langle A B C D\rangle= & \left(y_{2}-y_{1}\right)\left(x_{1} w_{2}-x_{2} w_{1}\right)\langle 1234\rangle+\left(z_{2}-z_{1}\right)\left(x_{1} w_{2}-x_{2} w_{1}\right)\langle 1235\rangle \\
& +\left(z_{1} y_{2}-z_{2} y_{1}\right)\left\{\langle 1245\rangle\left(x_{2}-x_{1}\right)+\langle 1345\rangle\left(w_{2}-w_{1}\right)+\langle 2345\rangle\left(x_{1} w_{2}-x_{2} w_{1}\right)\right\}
\end{aligned}
$$

When $\left(x_{1} w_{2}-x_{2} w_{1}\right)>0,\left(z_{1} y_{2}-z_{2} y_{1}\right)>0$, from $\langle A B C D\rangle$,

$$
y_{2}>y_{1}-a
$$

For

$$
\begin{aligned}
a= & \frac{\left(z_{2}-z_{1}\right)\left(x_{1} w_{2}-x_{2} w_{1}\right)\langle 1235\rangle}{\left(x_{1} w_{2}-x_{2} w_{1}\right)\langle 1234\rangle} \\
& +\frac{\left(z_{1} y_{2}-z_{2} y_{1}\right)\left\{\langle 1245\rangle\left(x_{2}-x_{1}\right)+\langle 1345\rangle\left(w_{2}-w_{1}\right)+\langle 2345\rangle\left(x_{1} w_{2}-x_{2} w_{1}\right)\right.}{\left(x_{1} w_{2}-x_{2} w_{1}\right)\langle 1234\rangle}
\end{aligned}
$$

The region of $y_{2}$ is depends on the sign of $a$. When $a<0$,

$$
\begin{aligned}
y_{2} & >y_{1}-a \\
z_{2} & <z_{1}-\frac{\left(z_{1} y_{2}-z_{2} y_{1}\right)\left\{\langle 1245\rangle\left(x_{2}-x_{1}\right)+\langle 1345\rangle\left(w_{2}-w_{1}\right)+\langle 2345\rangle\left(x_{1} w_{2}-x_{2} w_{1}\right)\right\}}{\left(x_{1} w_{2}-x_{2} w_{1}\right)\langle 1235\rangle} \\
& =z_{1}-b
\end{aligned}
$$

Similarly, the region of $z_{2}$ is depends on the sign of $b$. When $b<0$,

$$
z_{2}<z_{1}-b \text { and } x_{2}<x_{1}-\frac{\langle 1345\rangle\left(w_{2}-w_{1}\right)+\langle 2345\rangle\left(x_{1} w_{2}-x_{2} w_{1}\right)}{\langle 1245\rangle}=x_{1}-c
$$

When $c<0$,

$$
x_{2}<x_{1}-c \text { and } w_{2}<w_{1}-\frac{\langle 2345\rangle\left(x_{1} w_{2}-x_{2} w_{1}\right)}{\langle 1345\rangle}=w_{1}-d
$$


From $w_{2}>0,\left(x_{1} w_{2}-x_{2} w_{1}\right)>0$, then $d>0$ and there are 8 cases depending on the signs of a,b,c.

$$
\begin{aligned}
& \Omega_{1}=\frac{1}{x_{1}-\frac{w_{1}}{w_{2}} x_{2}}\left(\frac{1}{x_{2}}-\frac{1}{x_{2}-x_{1}+c}\right) \frac{1}{w_{1}-w_{2}-d} \frac{1}{w_{2}} \frac{1}{y_{1}} \frac{1}{y_{2}-y_{1}+a} \frac{1}{z_{1}-\frac{y_{1}}{y_{2}} z_{2}}\left(\frac{1}{z_{2}}-\frac{1}{z_{2}-z_{1}+c}\right) \\
& \Omega_{2}=\frac{1}{x_{1}-x_{2}-c}\left(\frac{1}{x_{2}}-\frac{1}{x_{2}-\frac{w_{2}}{w_{1}} x_{1}}\right)\left(\frac{1}{w_{1}}-\frac{1}{w_{1}-w_{2}-d}\right) \frac{1}{w_{2}} \frac{1}{y_{1}} \frac{1}{y_{2}-y_{1}+a} \\
& \times \frac{1}{z_{1}-\frac{y_{1}}{y_{2}} z_{2}}\left(\frac{1}{z_{2}}-\frac{1}{z_{2}-z_{1}+b}\right) \\
& \Omega_{3}=\frac{1}{x_{1}-\frac{w_{1}}{w_{2}} x_{2}} \frac{1}{x_{2}-x_{1}+c} \frac{1}{w_{1}-w_{2}-d} \frac{1}{w_{2}} \frac{1}{y_{1}} \frac{1}{y_{2}-y_{1}+a} \frac{1}{z_{1}-z_{2}-b}\left(\frac{1}{z_{2}}-\frac{1}{z_{2}-\frac{y_{2}}{y_{1}} z_{1}}\right) \\
& \Omega_{4}=\left(\frac{1}{x_{1}}-\frac{1}{x_{1}-x_{2}-c}\right)\left(\frac{1}{x_{2}}-\frac{1}{x_{2}-\frac{w_{2}}{w_{1}} x_{1}}\right)\left(\frac{1}{w_{1}}-\frac{1}{w_{1}-w_{2}-d}\right) \frac{1}{w_{2}} \frac{1}{y_{1}} \frac{1}{y_{2}-y_{1}+a} \\
& \times \frac{1}{z_{1}-z_{2}-b}\left(\frac{1}{z_{2}}-\frac{1}{z_{2}-\frac{y_{2}}{y_{1}} z_{1}}\right) \\
& \Omega_{5}=\frac{1}{x_{1}-\frac{w_{1}}{w_{2}} x_{2}}\left(\frac{1}{x_{2}}-\frac{1}{x_{2}-x_{1}+c}\right) \frac{1}{w_{1}-w_{2}-d} \frac{1}{w_{2}}\left(\frac{1}{y_{1}}-\frac{1}{y_{1}-y_{2}-a}\right) \frac{1}{y_{2}} \frac{1}{z_{1}-\frac{y_{1}}{y_{2}} z_{2}} \frac{1}{z_{2}-z_{1}+b} \\
& \Omega_{6}=\frac{1}{x_{1}-x_{2}-c}\left(\frac{1}{x_{2}}-\frac{1}{x_{2}-\frac{w_{2}}{w_{1}} x_{1}}\right)\left(\frac{1}{w_{1}}-\frac{1}{w_{1}-w_{2}-d}\right) \frac{1}{w_{2}}\left(\frac{1}{y_{1}}-\frac{1}{y_{1}-y_{2}-a}\right) \frac{1}{y_{2}} \\
& \times \frac{1}{z_{1}-\frac{y_{1}}{y_{2}} z_{2}} \frac{1}{z_{2}-z_{1}+b} \\
& \Omega_{7}=\frac{1}{x_{1}-\frac{w_{1}}{w_{2}} x_{2}} \frac{1}{x_{2}-x_{1}+c} \frac{1}{w_{1}-w_{2}-d} \frac{1}{w_{2}}\left(\frac{1}{y_{1}}-\frac{1}{y_{1}-y_{2}-a}\right) \frac{1}{y_{2}} \\
& \times\left(\frac{1}{z_{1}}-\frac{1}{z_{1}-z_{2}-b}\right)\left(\frac{1}{z_{2}}-\frac{1}{z_{2}-\frac{y_{2}}{y_{1}} z_{1}}\right) \\
& \Omega_{8}=\left(\frac{1}{x_{1}}-\frac{1}{x_{1}-x_{2}-c}\right)\left(\frac{1}{x_{2}}-\frac{1}{x_{2}-\frac{w_{2}}{w_{1}} x_{1}}\right)\left(\frac{1}{w_{1}}-\frac{1}{w_{1}-w_{2}-d}\right) \frac{1}{w_{2}}\left(\frac{1}{y_{1}}-\frac{1}{y_{1}-y_{2}-a}\right) \frac{1}{y_{2}} \\
& \times\left(\frac{1}{z_{1}}-\frac{1}{z_{1}-z_{2}-b}\right)\left(\frac{1}{z_{2}}-\frac{1}{z_{2}-\frac{y_{2}}{y_{1}} z_{1}}\right)
\end{aligned}
$$

For

$$
\begin{aligned}
a= & \frac{\left(z_{2}-z_{1}\right)\left(x_{1} w_{2}-x_{2} w_{1}\right)\langle 1235\rangle}{\left(x_{1} w_{2}-x_{2} w_{1}\right)\langle 1234\rangle} \\
& +\frac{\left(z_{1} y_{2}-z_{2} y_{1}\right)\left\{\langle 1245\rangle\left(x_{2}-x_{1}\right)+\langle 1345\rangle\left(w_{2}-w_{1}\right)+\langle 2345\rangle\left(x_{1} w_{2}-x_{2} w_{1}\right)\right.}{\left(x_{1} w_{2}-x_{2} w_{1}\right)\langle 1234\rangle} \\
b= & \frac{\left(z_{1} y_{2}-z_{2} y_{1}\right)\left\{\langle 1245\rangle\left(x_{2}-x_{1}\right)+\langle 1345\rangle\left(w_{2}-w_{1}\right)+\langle 2345\rangle\left(x_{1} w_{2}-x_{2} w_{1}\right)\right\}}{\left(x_{1} w_{2}-x_{2} w_{1}\right)\langle 1235\rangle} \\
c= & \frac{\langle 1345\rangle\left(w_{2}-w_{1}\right)+\langle 2345\rangle\left(x_{1} w_{2}-x_{2} w_{1}\right)}{\langle 1245\rangle} \\
d= & \frac{\langle 2345\rangle\left(x_{1} w_{2}-x_{2} w_{1}\right)}{\langle 1345\rangle}
\end{aligned}
$$


This is the case of $\left(x_{1} w_{2}-x_{2} w_{1}\right)>0,\left(z_{1} y_{2}-z_{2} y_{1}\right)>0$. Next we consider the case of $\left(x_{1} w_{2}-x_{2} w_{1}\right)>0,\left(z_{1} y_{2}-z_{2} y_{1}\right)<0$. Forms are obtained by replacement as follows.

$$
\begin{aligned}
\left(\frac{1}{x_{1}}-\frac{1}{x_{1}-x_{2}-c}\right) & \leftrightarrow \frac{1}{x_{1}-x_{2}-c} \\
\left(\frac{1}{x_{2}}-\frac{1}{x_{2}-x_{1}+c}\right) & \leftrightarrow \frac{1}{x_{2}-x_{1}+c} \\
\frac{1}{z_{1}-\frac{y_{1}}{y_{2}} z_{2}} & \rightarrow\left(\frac{1}{z_{1}}-\frac{1}{z_{1}-\frac{y_{1}}{y_{2}} z_{2}}\right) \\
\left(\frac{1}{z_{2}}-\frac{1}{z_{2}-\frac{y_{2}}{y_{1}} z_{1}}\right) & \rightarrow \frac{1}{z_{2}-\frac{y_{2}}{y_{1}} z_{1}}
\end{aligned}
$$

Then the cases of $\left(x_{1} w_{2}-x_{2} w_{1}\right)<0,\left(z_{1} y_{2}-z_{2} y_{1}\right)<0$ and $\left(x_{1} w_{2}-x_{2} w_{1}\right)<0,\left(z_{1} y_{2}-\right.$ $\left.z_{2} y_{1}\right)>0$ are obtained that swap $1 \leftrightarrow 2$ for the case of $\left(x_{1} w_{2}-x_{2} w_{1}\right)>0,\left(z_{1} y_{2}-z_{2} y_{1}\right)>0$ and $\left(x_{1} w_{2}-x_{2} w_{1}\right)>0,\left(z_{1} y_{2}-z_{2} y_{1}\right)<0$. Then sum of these 32 forms is

$$
\begin{aligned}
\Omega_{2424}= & \frac{d x_{1} d x_{2} \cdots d z_{1} d z_{2}}{x_{1} x_{2} w_{1} w_{2} y_{1} y_{2} z_{1} z_{2}} \frac{1}{\langle A B C D\rangle} \\
& \times\left\{\langle 1234\rangle\left(x_{2} w_{1} y_{1}+x_{1} w_{2} y_{2}\right)+y_{2} z_{1}\left(\langle 1345\rangle w_{2}+\langle 1245\rangle x_{2}+\langle 2345\rangle x_{1} w_{2}\right)\right. \\
& \left.+y_{1} z_{2}\left(\langle 1345\rangle w_{1}+\langle 1245\rangle x_{1}+\langle 2345\rangle x_{2} w_{1}\right)+\langle 1235\rangle\left(x_{2} w_{1} z_{1}+x_{1} w_{2} z_{2}\right)\right\}
\end{aligned}
$$

In the momentum twistor space,

$$
\begin{aligned}
& \Omega_{2424}=\frac{\left\langle 123 A_{4}\right\rangle\left\langle 123 C_{4}\right\rangle\left\langle A B d^{2} A\right\rangle\left\langle A B d^{2} B\right\rangle\left\langle C D d^{2} C\right\rangle\left\langle C D d^{2} D\right\rangle}{\left\{\begin{array}{c}
\langle A B 12\rangle\langle A B 13\rangle\langle A B 14\rangle\langle A B 15\rangle\langle A B 23\rangle\langle A B 45\rangle\langle A B C D\rangle \\
\times\langle C D 12\rangle\langle C D 13\rangle\langle C D 14\rangle\langle C D 15\rangle\langle C D 23\rangle\langle C D 45\rangle
\end{array}\right\}} \\
& \times\left\{\left\langle 123 A_{4}\right\rangle(\langle A B 12\rangle\langle C D 13\rangle\langle C D 45\rangle+\langle A B 15\rangle\langle C D 14\rangle\langle C D 23\rangle)\right. \\
& +\left\langle 123 C_{4}\right\rangle(\langle A B 13\rangle\langle A B 45\rangle\langle C D 12\rangle+\langle A B 14\rangle\langle A B 23\rangle\langle C D 15\rangle) \\
& +\langle 2345\rangle(\langle A B 12\rangle\langle A B 15\rangle\langle C D 13\rangle\langle C D 14\rangle+\langle A B 13\rangle\langle A B 14\rangle\langle C D 12\rangle\langle C D 15\rangle)\}
\end{aligned}
$$

The remaining patterns are $(3,4) \times(2,3),(2,4) \times(2,3),(3,4) \times(2,4)$. These forms can be obtained from $\Omega_{2334}, \Omega_{2324}, \Omega_{2434}$ that swap $A B \leftrightarrow C D$. 


\section{B Explicit results of the 2-loop n-point MHV amplituhedron}

First we consider the (1) case $i<k<l<j$,

$$
\begin{aligned}
& \langle A B C D\rangle= \\
& x_{1} x_{2} y_{2}\langle 1 i k l\rangle+x_{1} x_{2} z_{2}\langle 1 i k l+1\rangle+w_{2} x_{1} y_{2}\langle 1 i k+1 l\rangle+w_{2} x_{1} z_{2}\langle 1 i k+1 l+1\rangle-x_{1}\left(x_{2} y_{1}\langle 1 i k j\rangle\right. \\
& +x_{2} z_{1}\langle 1 i k j+1\rangle+w_{2} y_{1}\langle 1 i k+1 j\rangle+w_{2} z_{1}\langle 1 i k+1 j+1\rangle+y_{1} y_{2}\langle 1 i l j\rangle+y_{2} z_{1}\langle 1 i l j+1\rangle \\
& \left.+y_{1} z_{2}\langle 1 i l+1 j\rangle+z_{1} z_{2}\langle 1 i l+1 j+1\rangle\right)+w_{1} x_{2} y_{2}\langle 1 i+1 k l\rangle+w_{1} x_{2} z_{2}\langle 1 i+1 k l+1\rangle \\
& -w_{2}\left(w_{1} y_{1}\langle 1 i+1 k+1 j\rangle+w_{1} z_{1}\langle 1 i+1 k+1 j+1\rangle\right)+w_{1} w_{2} y_{2}\langle 1 i+1 k+1 l\rangle+w_{1} w_{2} z_{2}\langle 1 i+1 k+1 l+1\rangle \\
& -w_{1}\left(x_{2} y_{1}\langle 1 i+1 k j\rangle+x_{2} z_{1}\langle 1 i+1 k j+1\rangle+y_{1} y_{2}\langle 1 i+1 l j\rangle+y_{2} z_{1}\langle 1 i+1 l j+1\rangle+y_{1} z_{2}\langle 1 i+1 l+1 j\rangle\right. \\
& \left.+z_{1} z_{2}\langle 1 i+1 l+1 j+1\rangle\right)+x_{2} y_{1} y_{2}\langle 1 k l j\rangle+x_{2} y_{2} z_{1}\langle 1 k l j+1\rangle+x_{2} y_{1} z_{2}\langle 1 k l+1 j\rangle+x_{2} z_{1} z_{2}\langle 1 k l+1 j+1\rangle \\
& +w_{2} y_{1} y_{2}\langle 1 k+1 l j\rangle+w_{2} y_{2} z_{1}\langle 1 k+1 l j+1\rangle+w_{2} y_{1} z_{2}\langle 1 k+1 l+1 j\rangle+w_{2} z_{1} z_{2}\langle 1 k+1 l+1 j+1\rangle \\
& +x_{1} x_{2} y_{1} y_{2}\langle i k l j\rangle+x_{1} x_{2} y_{2} z_{1}\langle i k l j+1\rangle+x_{1} x_{2} y_{1} z_{2}\langle i k l+1 j\rangle+x_{1} x_{2} z_{1} z_{2}\langle i k l+1 j+1\rangle \\
& +w_{2} x_{1} y_{1} y_{2}\langle i k+1 l j\rangle+w_{2} x_{1} y_{2} z_{1}\langle i k+1 l j+1\rangle+w_{2} x_{1} y_{1} z_{2}\langle i k+1 l+1 j\rangle+w_{2} x_{1} z_{1} z_{2}\langle i k+1 l+1 j+1\rangle \\
& +w_{1} x_{2} y_{1} y_{2}\langle i+1 k l j\rangle+w_{1} x_{2} y_{2} z_{1}\langle i+1 k l j+1\rangle+w_{1} x_{2} y_{1} z_{2}\langle i+1 k l+1 j\rangle+w_{1} x_{2} z_{1} z_{2}\langle i+1 k l+1 j+1\rangle \\
& +w_{1} w_{2} y_{1} y_{2}\langle i+1 k+1 l j\rangle+w_{1} w_{2} y_{2} z_{1}\langle i+1 k+1 l j+1\rangle+w_{1} w_{2} y_{1} z_{2}\langle i+1 k+1 l+1 j\rangle \\
& +w_{1} w_{2} z_{1} z_{2}\langle i+1 k+1 l+1 j+1\rangle \\
& =a z_{2}-b w_{1}-c x_{1}-d w_{2}+e y_{2}
\end{aligned}
$$

for

$$
\begin{aligned}
a= & x_{1} x_{2}\langle 1 i k l+1\rangle+w_{2} x_{1}\langle 1 i k+1 l+1\rangle+w_{1} x_{2}\langle 1 i+1 k l+1\rangle+w_{1} w_{2}\langle 1 i+1 k+1 l+1\rangle+x_{2} y_{1}\langle 1 k l+1 j\rangle \\
& +x_{2} z_{1}\langle 1 k l+1 j+1\rangle+w_{2} y_{1}\langle 1 k+1 l+1 j\rangle+w_{2} z_{1}\langle 1 k+1 l+1 j+1\rangle+x_{1} x_{2} y_{1}\langle i k l+1 j\rangle \\
& +x_{1} x_{2} z_{1}\langle i k l+1 j+1\rangle+w_{2} x_{1} y_{1}\langle i k+1 l+1 j\rangle+w_{2} x_{1} z_{1}\langle i k+1 l+1 j+1\rangle+w_{1} x_{2} y_{1}\langle i+1 k l+1 j\rangle \\
& +w_{1} x_{2} z_{1}\langle i+1 k l+1 j+1\rangle+w_{1} w_{2} y_{1}\langle i+1 k+1 l+1 j\rangle+w_{1} w_{2} z_{1}\langle i+1 k+1 l+1 j+1\rangle \\
b= & x_{2} y_{1}\langle 1 i+1 k j\rangle+x_{2} z_{1}\langle 1 i+1 k j+1\rangle+y_{1} y_{2}\langle 1 i+1 l j\rangle+y_{2} z_{1}\langle 1 i+1 l j+1\rangle+y_{1} z_{2}\langle 1 i+1 l+1 j\rangle \\
& +z_{1} z_{2}\langle 1 i+1 l+1 j+1\rangle \\
c= & x_{2} y_{1}\langle 1 i k j\rangle+x_{2} z_{1}\langle 1 i k j+1\rangle+w_{2} y_{1}\langle 1 i k+1 j\rangle+w_{2} z_{1}\langle 1 i k+1 j+1\rangle+y_{1} y_{2}\langle 1 i l j\rangle+y_{2} z_{1}\langle 1 i l+1 j\rangle \\
& +y_{1} z_{2}\langle 1 i l+1 j\rangle+z_{1} z_{2}\langle 1 i l+1 j+1\rangle \\
d= & w_{1} y_{1}\langle 1 i+1 k+1 j\rangle+w_{1} z_{1}\langle 1 i+1 k+1 j+1\rangle \\
e= & x_{1} x_{2}\langle 1 i k l\rangle+w_{2} x_{1}\langle 1 i k+1 l\rangle+w_{1} x_{2}\langle 1 i+1 k l\rangle+w_{1} w_{2}\langle 1 i+1 k+1 l\rangle+x_{2} y_{1}\langle 1 k l j\rangle \\
& +x_{2} z_{1}\langle 1 k l j+1\rangle+w_{2} y_{1}\langle 1 k+1 l j\rangle+w_{2} z_{1}\langle 1 k+1 l j+1\rangle+x_{1} x_{2} y_{1}\langle i k l j\rangle+x_{1} x_{2} z_{1}\langle i k l j+1\rangle \\
& +w_{2} x_{1} y_{1}\langle i k+1 l j\rangle+w_{2} x_{1} z_{1}\langle i k+1 l j+1\rangle+w_{1} x_{2} y_{1}\langle i+1 k l j\rangle+w_{1} x_{2} z_{1}\langle i+1 k l j+1\rangle \\
& +w_{1} w_{2} y_{1}\langle i+1 k+1 l j\rangle+w_{1} w_{2} z_{1}\langle i+1 k+1 l j+1\rangle
\end{aligned}
$$

and $a, b, c, d, e>0$. From $\langle A B C D\rangle>0$,

$$
z_{2}>\frac{b}{a} w_{1}+\frac{c x_{1}+d w_{2}-e y_{2}}{a}
$$

In the case of $c x_{1}+d w_{2}-e y_{2}>0$,

$$
z_{2}>\frac{b}{a} w_{1}+\frac{c x_{1}+d w_{2}-e y_{2}}{a}, \text { and } y_{2}<\frac{c x_{1}+d w_{2}}{e}
$$


Then the form of this sign pattern is

$$
\Omega_{1}=\frac{1}{x_{1}} \frac{1}{x_{2}} \frac{1}{w_{1}} \frac{1}{w_{2}} \frac{1}{y_{1}}\left(\frac{1}{y_{2}}-\frac{1}{y_{2}-\frac{c x_{1}+d w_{2}}{e}}\right) \frac{1}{z_{1}} \frac{1}{z_{2}-\left(\frac{b}{a} w_{1}+\frac{c x_{1}+d w_{2}-e y_{2}}{a}\right)}
$$

Another pattern is that $c x_{1}+d w_{2}-e y_{2}<0$,

$$
w_{1}<\frac{a}{b} z_{2}-\frac{c x_{1}+d w_{2}-e y_{2}}{b}, \text { and } y_{2}>\frac{c x_{1}+d w_{2}}{e}
$$

Then the form is

$$
\Omega_{2}=\frac{1}{x_{1}} \frac{1}{x_{2}}\left(\frac{1}{w_{1}}-\frac{1}{w_{1}-\left(\frac{a}{b} z_{2}-\frac{c x_{1}+d w_{2}-e y_{2}}{b}\right)}\right) \frac{1}{w_{2}} \frac{1}{y_{1}} \frac{1}{y_{2}-\frac{c x_{1}+d w_{2}}{e}} \frac{1}{z_{1}} \frac{1}{z_{2}}
$$

The canonical form for this sign flip pattern is

$$
\begin{aligned}
\left(\Omega_{1}+\right. & \left.\Omega_{2}\right) d x_{1} d x_{2} \cdots d z_{1} d z_{2}=\frac{d x_{1} d x_{2} \cdots d z_{1} d z_{2}}{x_{1} x_{2} w_{1} w_{2} y_{1} y_{2} z_{1} z_{2}} \frac{1}{\left(a z_{2}-b w_{1}-c x_{1}-d w_{2}+e y_{2}\right)} \times \omega_{i j k l}^{1} \\
\omega_{i j k l}^{1} & =\langle 1 i k l\rangle x_{1} x_{2} y_{2}+\langle 1 i k l+1\rangle x_{1} x_{2} z_{2}+\langle 1 i k+1 l\rangle w_{2} x_{1} y_{2}+\langle 1 i k+1 l+1\rangle w_{2} x_{1} z_{2} \\
& +\langle 1 i+1 k l\rangle w_{1} x_{2} y_{2}+\langle 1 i+1 k l+1\rangle w_{1} x_{2} z_{2}+\langle 1 i+1 k+1 l\rangle w_{1} w_{2} y_{2}+\langle 1 i+1 k+1 l+1\rangle w_{1} w_{2} z_{2} \\
& +\langle 1 k l j\rangle x_{2} y_{1} y_{2}+\langle 1 k l j+1\rangle x_{2} y_{2} z_{1}+\langle 1 k l+1 j\rangle x_{2} y_{1} z_{2}+\langle 1 k l+1 j+1\rangle x_{2} z_{1} z_{2} \\
& +\langle 1 k+1 l j\rangle w_{2} y_{1} y_{2}+\langle 1 k+1 l j+1\rangle w_{2} y_{2} z_{1}+\langle 1 k+1 l+1 j\rangle w_{2} y_{1} z_{2} \\
& +\langle 1 k+1 l+1 j+1\rangle w_{2} z_{1} z_{2}+\langle i k l j\rangle x_{1} x_{2} y_{1} y_{2}+\langle i k l j+1\rangle x_{1} x_{2} y_{2} z_{1}+\langle i k l+1 j\rangle x_{1} x_{2} y_{1} z_{2} \\
& +\langle i k l+1 j+1\rangle x_{1} x_{2} z_{1} z_{2}+\langle i k+1 l j\rangle w_{2} x_{1} y_{1} y_{2}+\langle i k+1 l j+1\rangle w_{2} x_{1} z_{1} y_{2} \\
& +\langle i k+1 l+1 j\rangle w_{2} x_{1} y_{1} z_{2}+\langle i k+1 l+1 j+1\rangle w_{2} x_{1} z_{1} z_{2}+\langle i+1 k+1 l j\rangle w_{2} w_{1} y_{1} y_{2} \\
& +\langle i+1 k+1 l j+1\rangle w_{2} w_{1} z_{1} y_{2}+\langle i+1 k+1 l+1 j\rangle w_{2} w_{1} y_{1} z_{2}+\langle i+1 k+1 l+1 j+1\rangle w_{2} w_{1} z_{1} z_{2} \\
& +\langle i+1 k l j\rangle w_{1} x_{2} y_{1} y_{2}+\langle i+1 k l j+1\rangle w_{1} x_{2} y_{2} z_{1}+\langle i+1 k l+1 j\rangle w_{1} x_{2} y_{1} z_{2} \\
& +\langle i+1 k l+1 j+1\rangle w_{1} x_{2} z_{1} z_{2}
\end{aligned}
$$

In the momentum twistor space,

$$
\Omega_{i j k l}^{1}=\frac{\left\langle 1 i i+1 A_{j}\right\rangle\left\langle 1 k k+1 C_{l}\right\rangle\left\langle A B d^{2} A\right\rangle\left\langle A B d^{2} B\right\rangle\left\langle C D d^{2} C\right\rangle\left\langle C D d^{2} D\right\rangle}{\langle A B 1 i\rangle\langle A B 1 i+1\rangle\langle A B 1 j\rangle\langle A B 1 j+1\rangle\langle A B C D\rangle\langle C D 1 k\rangle\langle C D 1 k+1\rangle\langle C D 1 l\rangle\langle C D 1 l+1\rangle} \times \omega_{i j k l}^{1^{\prime}} .
$$

for

$$
\omega_{i j k l}^{1^{\prime}}=\frac{\langle A B i i+1\rangle\left\langle A_{j} C_{k} C_{l} 1\right\rangle+\left\langle A_{i} A_{j} C_{k} C_{l}\right\rangle}{\langle A B i i+1\rangle\langle A B j j+1\rangle\langle C D k k+1\rangle\langle C D l l+1\rangle} .
$$

Another forms can be obtained similarly

$$
\begin{aligned}
\omega_{i j k l}^{2}= & \langle 1 i k l\rangle x_{1} x_{2} y_{2}+\langle 1 i k l+1\rangle x_{1} x_{2} z_{2}+\langle 1 i k+1 l\rangle w_{2} x_{1} y_{2}+\langle 1 i k+1 l+1\rangle w_{2} x_{1} z_{2}+\langle 1 i j l\rangle x_{1} y_{1} y_{2} \\
& +\langle 1 i j l+1\rangle x_{1} y_{1} z_{2}+\langle 1 i j+1 l\rangle z_{1} x_{1} y_{2}+\langle 1 i j+1 l+1\rangle z_{1} x_{1} z_{2}+\langle 1 i+1 k l\rangle w_{1} x_{2} y_{2} \\
& +\langle 1 i+1 k l+1\rangle w_{1} x_{2} z_{2}+\langle 1 i+1 k+1 l\rangle w_{2} w_{1} y_{2}+\langle 1 i+1 k+1 l+1\rangle w_{2} w_{1} z_{2}+\langle 1 i+1 j l\rangle w_{1} y_{1} y_{2} \\
& +\langle 1 i+1 j l+1\rangle w_{1} y_{1} z_{2}+\langle 1 i+1 j+1 l\rangle z_{1} w_{1} y_{2}+\langle 1 i+1 j+1 l+1\rangle z_{1} w_{1} z_{2}
\end{aligned}
$$




$$
\begin{aligned}
& \omega_{i j k l}^{3}=\langle 1 i j k\rangle x_{1} x_{2} y_{1}+\langle 1 i j k+1\rangle w_{2} x_{1} y_{1}+\langle 1 i j l\rangle x_{1} y_{1} y_{2}+\langle 1 i j l+1\rangle x_{1} y_{1} z_{2}+\langle 1 i j+1 k\rangle x_{1} x_{2} z_{1} \\
& +\langle 1 i j+1 k+1\rangle w_{2} x_{1} z_{1}+\langle 1 i j+1 l\rangle x_{1} y_{2} z_{1}+\langle 1 i j+1 l+1\rangle x_{1} z_{1} z_{2}+\langle 1 i k l\rangle x_{1} x_{2} y_{2} \\
& +\langle 1 i k l+1\rangle x_{1} x_{2} z_{2}+\langle 1 i k+1 l\rangle w_{2} x_{1} y_{2}+\langle 1 i k+1 l+1\rangle w_{2} x_{1} z_{2}+\langle 1 i+1 j k\rangle w_{1} x_{2} y_{1} \\
& +\langle 1 i+1 j k+1\rangle w_{2} w_{1} y_{1}+\langle 1 i+1 j l\rangle w_{1} y_{1} y_{2}+\langle 1 i+1 j l+1\rangle w_{1} y_{1} z_{2}+\langle 1 i+1 j+1 k\rangle w_{1} x_{2} z_{1} \\
& +\langle 1 i+1 j+1 k+1\rangle w_{2} w_{1} z_{1}+\langle 1 i+1 j+1 l\rangle w_{1} y_{2} z_{1}+\langle 1 i+1 j+1 l+1\rangle w_{1} z_{1} z_{2} \\
& +\langle 1 i+1 k l\rangle w_{1} x_{2} y_{2}+\langle 1 i+1 k l+1\rangle w_{1} x_{2} z_{2}+\langle 1 i+1 k+1 l\rangle w_{2} w_{1} y_{2}+\langle 1 i+1 k+1 l+1\rangle w_{2} w_{1} z_{2} \\
& +\langle 1 j k l\rangle x_{2} y_{1} y_{2}+\langle 1 j k l+1\rangle x_{2} y_{1} z_{2}+\langle 1 j k+1 l\rangle w_{2} y_{1} y_{2}+\langle 1 j k+1 l+1\rangle w_{2} y_{1} z_{2}+\langle 1 j+1 k l\rangle x_{2} z_{1} y_{2} \\
& +\langle 1 j+1 k l+1\rangle x_{2} z_{1} z_{2}+\langle 1 j+1 k+1 l\rangle w_{2} z_{1} y_{2}+\langle 1 j+1 k+1 l+1\rangle w_{2} z_{1} z_{2} \\
& +\langle i j k l\rangle x_{1} x_{2} y_{1} y_{2}+\langle i j k l+1\rangle x_{1} x_{2} y_{1} z_{2}+\langle i j k+1 l\rangle w_{2} x_{1} y_{1} y_{2}+\langle i j k+1 l+1\rangle w_{2} x_{1} y_{1} z_{2} \\
& +\langle i j+1 k l\rangle x_{1} x_{2} z_{1} y_{2}+\langle i j+1 k l+1\rangle x_{1} x_{2} z_{1} z_{2}+\langle i j+1 k+1 l\rangle w_{2} x_{1} z_{1} y_{2} \\
& +\langle i j+1 k+1 l+1\rangle w_{2} x_{1} z_{1} z_{2}+\langle i+1 j k l\rangle w_{1} x_{2} y_{1} y_{2}+\langle i+1 j k l+1\rangle w_{1} x_{2} y_{1} z_{2} \\
& +\langle i+1 j k+1 l\rangle w_{2} w_{1} y_{1} y_{2}+\langle i+1 j k+1 l+1\rangle w_{2} w_{1} y_{1} z_{2}+\langle i+1 j+1 k l\rangle w_{1} x_{2} z_{1} y_{2} \\
& +\langle i+1 j+1 k l+1\rangle w_{1} x_{2} z_{1} z_{2}+\langle i+1 j+1 k+1 l\rangle w_{2} w_{1} z_{1} y_{2}+\langle i+1 j+1 k+1 l+1\rangle w_{2} w_{1} z_{1} z_{2} \\
& \omega_{i j k l}^{4}=\langle 1 i i+1 l\rangle w_{2} x_{1} y_{2}+\langle 1 i i+1 l+1\rangle w_{2} x_{1} z_{2}+\langle 1 i i+1 j\rangle w_{1} x_{2} y_{1}+\langle 1 i i+1 j+1\rangle w_{1} x_{2} z_{1} \\
& +\langle 1 i l j\rangle x_{2} y_{1} y_{2}+\langle 1 i l j+1\rangle x_{2} y_{2} z_{1}+\langle 1 i l+1 j\rangle x_{2} y_{1} z_{2}+\langle 1 i l+1 j+1\rangle x_{2} z_{1} z_{2}+\langle 1 i+1 l j\rangle w_{2} y_{1} y_{2} \\
& +\langle 1 i+1 l j+1\rangle w_{2} y_{2} z_{1}+\langle 1 i+1 l+1 j\rangle w_{2} y_{1} z_{2}+\langle 1 i+1 l+1 j+1\rangle w_{2} z_{1} z_{2}+\langle i i+1 l j\rangle w_{2} x_{1} y_{1} y_{2} \\
& +\langle i i+1 l j+1\rangle w_{2} x_{1} y_{2} z_{1}+\langle i i+1 l+1 j\rangle w_{2} x_{1} y_{1} z_{2}+\langle i i+1 l+1 j+1\rangle w_{2} x_{1} z_{1} z_{2} \\
& \omega_{i j k l}^{5}=\langle 1 i i+1 j\rangle\left(w_{1} x_{2} y_{1}+w_{2} x_{1} y_{2}\right)+\langle 1 i i+1 j+1\rangle\left(w_{1} x_{2} z_{1}+w_{2} x_{1} z_{2}\right)+\langle 1 i j j+1\rangle\left(x_{2} y_{2} z_{1}+x_{1} y_{1} z_{2}\right) \\
& +\langle 1 i+1 j j+1\rangle\left(w_{2} y_{2} z_{1}+w_{1} y_{1} z_{2}\right)+\langle i i+1 j j+1\rangle\left(w_{2} x_{1} y_{2} z_{1}+w_{1} x_{2} y_{1} z_{2}\right) \\
& \omega_{i j k l}^{6}=\langle 1 i k j\rangle x_{1} x_{2} y_{2}+\langle 1 i k j+1\rangle x_{1} x_{2} z_{2}+\langle 1 i k+1 j\rangle w_{2} x_{1} y_{2}+\langle 1 i k+1 j+1\rangle w_{2} x_{1} z_{2}+\langle 1 i j j+1\rangle x_{1} y_{1} z_{2} \\
& +\langle 1 i+1 k j\rangle w_{1} x_{2} y_{2}+\langle 1 i+1 k j+1\rangle w_{1} x_{2} z_{2}+\langle 1 i+1 k+1 j\rangle w_{2} w_{1} y_{2}+\langle 1 i+1 k+1 j+1\rangle w_{2} w_{1} z_{2} \\
& +\langle 1 i+1 j j+1\rangle w_{1} y_{1} z_{2}+\langle 1 k j j+1\rangle x_{2} y_{2} z_{1}+\langle 1 k+1 j j+1\rangle w_{2} y_{2} z_{1}+\langle i k j j+1\rangle x_{1} x_{2} y_{2} z_{1} \\
& +\langle i k+1 j j+1\rangle w_{2} x_{1} y_{2} z_{1}+\langle i+1 k j j+1\rangle w_{1} x_{2} y_{2} z_{1}+\langle i+1 k+1 j j+1\rangle w_{1} w_{2} y_{2} z_{1} \\
& \omega_{i j k l}^{7}=\langle 1 i j j+1\rangle w_{2} x_{1} y_{1}+\langle 1 i j l\rangle\left(x_{1} x_{2} y_{2}+x_{1} y_{1} y_{2}\right)+\langle 1 i j l+1\rangle\left(x_{1} x_{2} z_{2}+x_{1} y_{1} z_{2}\right) \\
& +\langle 1 i j+1 l\rangle\left(w_{2} x_{1} y_{2}+x_{1} y_{2} z_{1}\right)+\langle 1 i j+1 l+1\rangle\left(w_{2} x_{1} z_{2}+x_{1} z_{1} z_{2}\right)+\langle 1 i+1 j j+1\rangle w_{2} w_{1} y_{1} \\
& +\langle 1 i+1 j l\rangle\left(w_{1} x_{2} y_{2}+w_{1} y_{1} y_{2}\right)+\langle 1 i+1 j l+1\rangle\left(w_{1} x_{2} z_{2}+w_{1} y_{1} z_{2}\right) \\
& +\langle 1 i+1 j+1 l\rangle\left(w_{2} w_{1} y_{2}+w_{1} y_{2} z_{1}\right)+\langle 1 i+1 j+1 l+1\rangle\left(w_{2} w_{1} z_{2}+w_{1} z_{1} z_{2}\right)+\langle 1 j j+1 l\rangle w_{2} y_{1} y_{2} \\
& +\langle 1 j j+1 l+1\rangle w_{2} y_{1} z_{2}+\langle i j j+1 l\rangle w_{2} x_{1} y_{1} y_{2}+\langle i j j+1 l+1\rangle w_{2} x_{1} y_{1} z_{2}+\langle i+1 j j+1 l\rangle w_{1} w_{2} y_{1} y_{2} \\
& +\langle i+1 j j+1 l+1\rangle w_{1} w_{2} y_{1} z_{2}
\end{aligned}
$$

Next, we rewrite these forms in the momentum twistor space

$$
\begin{aligned}
\omega_{i j k l}^{1^{\prime}} & =\frac{\langle A B i i+1\rangle\left\langle A_{j} C_{k} C_{l} 1\right\rangle+\left\langle A_{i} A_{j} C_{k} C_{l}\right\rangle}{\langle A B i i+1\rangle\langle A B j j+1\rangle\langle C D k k+1\rangle\langle C D l l+1\rangle} . \\
\omega_{i j k l}^{2^{\prime}} & =\frac{-\langle A B j j+1\rangle\left\langle A_{i} C_{k} C_{l} 1\right\rangle+\langle C D k k+1\rangle\left\langle A_{i} A_{j} C_{l} 1\right\rangle}{\langle A B i i+1\rangle\langle A B j j+1\rangle\langle C D k k+1\rangle\langle C D l l+1\rangle}
\end{aligned}
$$




$$
\begin{aligned}
\omega_{i j k l}^{3^{\prime}}= & \frac{1}{\langle A B i i+1\rangle\langle A B j j+1\rangle\langle C D k k+1\rangle\langle C D l l+1\rangle} \\
& \times\left\{\langle A B i i+1\rangle\left\langle A_{j} C_{k} C_{l} 1\right\rangle-\langle A B j j+1\rangle\left\langle A_{i} C_{k} C_{l} 1\right\rangle-\langle A B 1 i\rangle\left\langle i+1 A_{j} C_{k} C_{l}\right\rangle\right. \\
& \left.+\langle C D k k+1\rangle\left\langle A_{i} A_{j} C_{l} 1\right\rangle-\langle C D l l+1\rangle\left\langle A_{i} A_{j} C_{k} 1\right\rangle+\langle A B 1 i+1\rangle\left\langle i A_{j} C_{k} C_{l}\right\rangle\right\} \\
\omega_{i j k l}^{4^{\prime}}= & \frac{1}{\langle A B i i+1\rangle\langle A B j j+1\rangle\langle C D i i+1\rangle\langle C D l l+1\rangle} \\
& \times\left\{\langle A B i i+1\rangle\left\langle A_{j} C_{k} C_{l} 1\right\rangle+\langle A B 1 i+1\rangle\langle C D 1 i\rangle\left\langle A_{j} C_{l} i i+1\right\rangle\right. \\
& \left.+\langle A B 1 i\rangle\langle C D 1 i+1\rangle\langle C D l l+1\rangle\left\langle A_{j} 1 i i+1\right\rangle+\langle A B 1 i+1\rangle\langle C D 1 i\rangle\langle A B j j+1\rangle\left\langle C_{l} 1 i i+1\right\rangle\right\} \\
\omega_{i j k l}^{5^{\prime}}= & \frac{1}{\langle A B i i+1\rangle\langle A B j j+1\rangle\langle C D i i+1\rangle\langle C D j j+1\rangle} \\
& \times\left\{\left\langle 1 i i+1 A_{j}\right\rangle(\langle A B 1 i\rangle\langle C D 1 k+1\rangle\langle C D l l+1\rangle+\langle A B 1 j+1\rangle\langle C D 1 l\rangle\langle C D k k+1\rangle)\right. \\
& +\left\langle 1 k k+1 C_{l}\right\rangle(\langle A B 1 i+1\rangle\langle A B j j+1\rangle\langle C D 1 k\rangle+\langle A B 1 j\rangle\langle A B i i+1\rangle\langle C D 1 l+1\rangle) \\
& +\langle i i+1 j j+1\rangle(\langle A B 1 i\rangle\langle A B 1 j+1\rangle\langle C D 1 k+1\rangle\langle C D 1 l\rangle+\langle A B 1 i+1\rangle\langle A B 1 j\rangle\langle C D 1 k\rangle\langle C D 1 l+1\rangle)\} \\
\omega_{i j k l}^{6^{\prime}}= & \frac{1}{\langle A B i i+1\rangle\langle A B j j+1\rangle\langle C D k k+1\rangle\langle C D j j+1\rangle} \\
& \times\left\{\langle A B 1 j\rangle\langle A B i i+1\rangle\langle C D 1 l+1\rangle\left\langle C_{k} 1 j j+1\right\rangle+\langle A B 1 j+1\rangle\langle C D 1 l\rangle\langle C D k k+1\rangle\left\langle A_{i} 1 j j+1\right\rangle\right. \\
& \left.+\langle A B j j+1\rangle\left(\langle C D 1 l+1\rangle\left\langle A_{i} C_{k} 1 j\right\rangle-\langle C D 1 l\rangle\left\langle A_{i} C_{k} 1 j+1\right\rangle\right)\right\} \\
\omega_{i j k l}^{7^{\prime}}= & \frac{1}{\langle A B i i+1\rangle\langle A B j j+1\rangle\langle C D j j+1\rangle\langle C D l l+1\rangle} \\
& \times\left\{\langle A B 1 j\rangle\langle C D 1 j+1\rangle\langle A B 1 i\rangle\left\langle i+1 j j+1 C_{k}\right\rangle-\langle A B 1 j\rangle\langle C D 1 j+1\rangle\langle A B 1 i+1\rangle\left\langle i j j+1 C_{k}\right\rangle\right. \\
& +\langle A B 1 j+1\rangle\langle C D 1 j\rangle\langle C D k k+1\rangle\left\langle 1 j j+1 A_{i}\right\rangle+\langle A B 1 j\rangle\langle C D 1 j+1\rangle\langle A B i i+1\rangle\left\langle 1 j j+1 C_{k}\right\rangle \\
& \left.+\langle A B j j+1\rangle\left\langle 1 C_{k} C_{j} A_{i}\right\rangle\right\} \\
\omega_{i j k l}^{8^{\prime}}= & \omega_{i j k l}^{4^{\prime}}, \quad \omega_{i j k l}^{9^{\prime}}=\omega_{i j k l}^{7^{\prime}}, \quad \omega_{i j k l}^{10^{\prime}}=\omega_{i j k l}^{2^{\prime}}, \quad \omega_{i j k l}^{11^{\prime}}=\omega_{i j k l}^{6^{\prime}}, \quad \omega_{i j k l}^{12^{\prime}}=\omega_{i j k l}^{1^{\prime}}, \quad \omega_{i j k l}^{13^{\prime}}=\omega_{i j k l}^{3^{\prime}}, \\
& (A B) \leftrightarrow(C D),(i, j) \leftrightarrow(k, l)
\end{aligned}
$$

\section{Explicit results of the 2-loop n-point MHV log amplituhedron}

$$
\Omega\left[\log \left[\mathcal{A}_{\mathrm{MHV}}^{n-\text { pt 2-loop }}\right]\right]=\sum_{\substack{i, j, k, l=2,3, \cdots, n-1 \\ i<k<l<j}} \Omega_{i j k l}^{1}[\log ]+\sum_{i<k<j<l} \Omega_{i j k l}^{2}[\log ] \cdots+\sum_{k<l<i<j} \Omega_{i j k l}^{13}[\log ]
$$

for

$$
\Omega_{i j k l}^{m}[\log ]=\frac{d x_{1} d x_{2} \cdots d z_{1} d z_{2}}{x_{1} x_{2} w_{1} w_{2} y_{1} y_{2} z_{1} z_{2}} \frac{-1}{\left(a z_{2}-b w_{1}-c x_{1}-d w_{2}+e y_{2}\right)} \times \omega_{i j k l}^{m}[\log ]
$$

where

$$
\begin{aligned}
\omega_{i j k l}^{1}[\log ]= & x_{2} y_{1}\langle 1 i+1 k j\rangle+x_{2} z_{1}\langle 1 i+1 k j+1\rangle+y_{1} y_{2}\langle 1 i+1 l j\rangle+y_{2} z_{1}\langle 1 i+1 l j+1\rangle \\
& +y_{1} z_{2}\langle 1 i+1 l+1 j\rangle+z_{1} z_{2}\langle 1 i+1 l+1 j+1\rangle+x_{2} y_{1}\langle 1 i k j\rangle+x_{2} z_{1}\langle 1 i k j+1\rangle \\
& +w_{2} y_{1}\langle 1 i k+1 j\rangle+w_{2} z_{1}\langle 1 i k+1 j+1\rangle+y_{1} y_{2}\langle 1 i l j\rangle+y_{2} z_{1}\langle 1 i l+1 j\rangle+y_{1} z_{2}\langle 1 i l+1 j\rangle \\
& +z_{1} z_{2}\langle 1 i l+1 j+1\rangle+w_{1} y_{1}\langle 1 i+1 k+1 j\rangle+w_{1} z_{1}\langle 1 i+1 k+1 j+1\rangle
\end{aligned}
$$




$$
\begin{aligned}
& \omega_{i j k l}^{2}[\log ]=-x_{1} x_{2} y_{1}\langle 1 i k j\rangle-x_{1} x_{2} z_{1}\langle 1 i k j+1\rangle-w_{2} x_{1} y_{1}\langle 1 i k+1 j\rangle \\
& -w_{2} x_{1} z_{1}\langle 1 i k+1 j+1\rangle-w_{1} x_{2} y_{1}\langle 1 i+1 k j\rangle-w_{1} x_{2} z_{1}\langle 1 i+1 k j+1\rangle-w_{1} w_{2} y_{1}\langle 1 i+1 k+1 j\rangle \\
& -w_{1} w_{2} z_{1}\langle 1 i+1 k+1 j+1\rangle-x_{2} y_{1} y_{2}\langle 1 k j l\rangle-x_{2} y_{1} z_{2}\langle 1 k j l+1\rangle-x_{2} y_{2} z_{1}\langle 1 k j+1 l\rangle \\
& -x_{2} z_{1} z_{2}\langle 1 k j+1 l+1\rangle-w_{2} y_{1} y_{2}\langle 1 k+1 j l\rangle-w_{2} y_{1} z_{2}\langle 1 k+1 j l+1\rangle-w_{2} y_{2} z_{1}\langle 1 k+1 j+1 l\rangle \\
& -w_{2} z_{1} z_{2}\langle 1 k+1 j+1 l+1\rangle-x_{1} x_{2} y_{1} y_{2}\langle i k j l\rangle-x_{1} x_{2} y_{1} z_{2}\langle i k j l+1\rangle-x_{1} x_{2} y_{2} z_{1}\langle i k j+1 l\rangle \\
& -x_{1} x_{2} z_{1} z_{2}\langle i k j+1 l+1\rangle-w_{2} x_{1} y_{1} y_{2}\langle i k+1 j l\rangle-w_{2} x_{1} y_{1} z_{2}\langle i k+1 j l+1\rangle \\
& -w_{2} x_{1} y_{2} z_{1}\langle i k+1 j+1 l\rangle-w_{2} x_{1} z_{1} z_{2}\langle i k+1 j+1 l+1\rangle-w_{1} x_{2} y_{1} y_{2}\langle i+1 k j l\rangle \\
& -w_{1} x_{2} y_{1} z_{2}\langle i+1 k j l+1\rangle-w_{1} x_{2} y_{2} z_{1}\langle i+1 k j+1 l\rangle-w_{1} x_{2} z_{1} z_{2}\langle i+1 k j+1 l+1\rangle \\
& -w_{1} w_{2} y_{1} y_{2}\langle i+1 k+1 j l\rangle-w_{1} w_{2} y_{1} z_{2}\langle i+1 k+1 j l+1\rangle-w_{1} w_{2} y_{2} z_{1}\langle i+1 k+1 j+1 l\rangle \\
& -w_{1} w_{2} z_{1} z_{2}\langle i+1 k+1 j+1 l+1\rangle \\
& \omega_{i j k l}^{3}[\log ]=0 \\
& \omega_{i j k l}^{4}[\log ]=-w_{1} x_{2} y_{2}\langle 1 i i+1 l\rangle-w_{1} x_{2} z_{2}\langle 1 i i+1 l+1\rangle-w_{2} x_{1} y_{1}\langle 1 i i+1 j\rangle-w_{2} x_{1} z_{1}\langle 1 i i+1 j+1\rangle \\
& -x_{1} y_{1} y_{2}\langle 1 i l j\rangle-x_{1} y_{2} z_{1}\langle 1 i l j+1\rangle-x_{1} y_{1} z_{2}\langle 1 i l+1 j\rangle-x_{1} z_{1} z_{2}\langle 1 i l+1 j+1\rangle-w_{1} y_{1} y_{2}\langle 1 i+1 l j\rangle \\
& -w_{1} y_{2} z_{1}\langle 1 i+1 l j+1\rangle-w_{1} y_{1} z_{2}\langle 1 i+1 l+1 j\rangle-w_{1} z_{1} z_{2}\langle 1 i+1 l+1 j+1\rangle-w_{1} x_{2} y_{1} y_{2}\langle i i+1 l j\rangle \\
& -w_{1} x_{2} y_{2} z_{1}\langle i i+1 l j+1\rangle-w_{1} x_{2} y_{1} z_{2}\langle i i+1 l+1 j\rangle-w_{1} x_{2} z_{1} z_{2}\langle i i+1 l+1 j+1\rangle \\
& -x_{1} y_{2} z_{1}\langle 1 i j j+1\rangle-x_{2} y_{1} z_{2}\langle 1 i j j+1\rangle-w_{1} y_{2} z_{1}\langle 1 i+1 j j+1\rangle-w_{2} y_{1} z_{2}\langle 1 i+1 j j+1\rangle \\
& -w_{1} x_{2} y_{2} z_{1}\langle i i+1 j j+1\rangle-w_{2} x_{1} y_{1} z_{2}\langle i i+1 j j+1\rangle
\end{aligned}
$$

$\left.\omega_{i j k l}^{8}[\log ]=\omega_{i j k l}^{4}[\log ], \quad \omega_{i j k l}^{9}[\log ]=\omega_{i j k l}^{7}[\log ], \quad \omega_{i j k l}^{10}[\log ]=\omega_{i j k l}^{2}[\log ], \quad \omega_{i j k l}^{11} \log \right]=\omega_{i j k l}^{6}[\log ]$,

$\omega_{i j k l}^{12}[\log ]=\omega_{i j k l}^{1}[\log ], \quad \omega_{i j k l}^{13}[\log ]=0$,

$\left(x_{1}, w_{1}, y_{1}, z_{1}\right) \leftrightarrow\left(x_{2}, w_{2}, y_{2}, z_{2}\right),(i, j) \leftrightarrow(k, l)$

We can similarly write these forms in the momentum twistor language.

Open Access. This article is distributed under the terms of the Creative Commons Attribution License (CC-BY 4.0), which permits any use, distribution and reproduction in any medium, provided the original author(s) and source are credited. 


\section{References}

[1] A. Hodges, Eliminating spurious poles from gauge-theoretic amplitudes, JHEP 05 (2013) 135 [arXiv:0905.1473] [INSPIRE].

[2] N. Arkani-Hamed, J.L. Bourjaily, F. Cachazo, A. Hodges and J. Trnka, A Note on Polytopes for Scattering Amplitudes, JHEP 04 (2012) 081 [arXiv: 1012.6030] [INSPIRE].

[3] N. Arkani-Hamed, J.L. Bourjaily, F. Cachazo, A.B. Goncharov, A. Postnikov and J. Trnka, Grassmannian Geometry of Scattering Amplitudes, Cambridge University Press (2016) [arXiv:1212.5605] [INSPIRE].

[4] N. Arkani-Hamed, Y. Bai and T. Lam, Positive Geometries and Canonical Forms, JHEP 11 (2017) 039 [arXiv: 1703.04541] [INSPIRE].

[5] N. Arkani-Hamed and J. Trnka, The Amplituhedron, JHEP 10 (2014) 030 [arXiv: 1312.2007] [INSPIRE].

[6] N. Arkani-Hamed and J. Trnka, Into the Amplituhedron, JHEP 12 (2014) 182 [arXiv: 1312.7878] [INSPIRE].

[7] N. Arkani-Hamed, H. Thomas and J. Trnka, Unwinding the Amplituhedron in Binary, JHEP 01 (2018) 016 [arXiv: 1704.05069] [INSPIRE].

[8] N. Arkani-Hamed, A. Hodges and J. Trnka, Positive Amplitudes In The Amplituhedron, JHEP 08 (2015) 030 [arXiv:1412.8478] [INSPIRE].

[9] L. Ferro, T. Lukowski, A. Orta and M. Parisi, Towards the Amplituhedron Volume, JHEP 03 (2016) 014 [arXiv: 1512.04954] [INSPIRE].

[10] L. Ferro, T. Łukowski and M. Parisi, Amplituhedron meets Jeffrey-Kirwan residue, J. Phys. A 52 (2019) 045201 [arXiv: 1805.01301] [INSPIRE].

[11] N. Arkani-Hamed, J.L. Bourjaily, F. Cachazo, S. Caron-Huot and J. Trnka, The All-Loop Integrand For Scattering Amplitudes in Planar N=4 SYM, JHEP 01 (2011) 041 [arXiv: 1008.2958] [INSPIRE].

[12] N. Arkani-Hamed, J.L. Bourjaily, F. Cachazo and J. Trnka, Local Integrals for Planar Scattering Amplitudes, JHEP 06 (2012) 125 [arXiv: 1012.6032] [INSPIRE].

[13] C. Chang, C. Monstein, A. Refregier, A. Amara, A. Glauser and S. Casura, Beam calibration of radio telescopes with drones, Publ. Astron. Soc. Pac. 127 (2015) 1131 [arXiv:1505.05885] [INSPIRE].

[14] N. Arkani-Hamed, C. Langer, A. Yelleshpur Srikant and J. Trnka, Deep Into the Amplituhedron: Amplitude Singularities at All Loops and Legs, Phys. Rev. Lett. 122 (2019) 051601 [arXiv: 1810.08208] [INSPIRE].

[15] S.N. Karp, L.K. Williams and Y.X. Zhang, Decompositions of amplituhedra, arXiv: 1708.09525 [INSPIRE].

[16] S.N. Karp and L.K. Williams, The $m=1$ amplituhedron and cyclic hyperplane arrangements, arXiv: 1608.08288 [INSPIRE].

[17] P. Heslop and A. Stewart, The twistor Wilson loop and the amplituhedron, JHEP 10 (2018) 142 [arXiv: 1807.05921] [INSPIRE].

[18] J. Rao, 4-particle Amplituhedronics for 3-5 loops, arXiv:1806.01765 [INSPIRE].

[19] P. Galashin and T. Lam, Parity duality for the amplituhedron, arXiv:1805.00600 [INSPIRE]. 
[20] Y. An, Y. Li, Z. Li and J. Rao, All-loop Mondrian Diagrammatics and 4-particle Amplituhedron, JHEP 06 (2018) 023 [arXiv:1712.09994] [INSPIRE].

[21] J. Rao, 4-particle Amplituhedron at 3-loop and its Mondrian Diagrammatic Implication, JHEP 06 (2018) 038 [arXiv: 1712.09990] [INSPIRE].

[22] D. Galloni, Positivity Sectors and the Amplituhedron, arXiv:1601.02639 [INSPIRE].

[23] S. Franco, D. Galloni, A. Mariotti and J. Trnka, Anatomy of the Amplituhedron, JHEP 03 (2015) 128 [arXiv:1408.3410] [INSPIRE].

[24] Y. Bai and S. He, The Amplituhedron from Momentum Twistor Diagrams, JHEP 02 (2015) 065 [arXiv: 1408.2459] [INSPIRE]. 